\title{
Transport phenomena and microscopic structure in partially miscible binary fluids: A simulation study of the symmetrical Lennard-Jones mixture
}

\author{
Subir K. Das, Jürgen Horbach, and Kurt Binder \\ Institut für Physik, Johannes Gutenberg-Universität \\ D-55099 Mainz, Staudinger Weg 7, Germany
}

\begin{abstract}
Static and dynamic structure factors and various transport coefficients are computed for a Lennard-Jones model of a binary fluid (A,B) with a symmetrical miscibility gap, varying both temperature and relative concentration of the mixture. The model is first equilibrated by a semi-grandcanonical Monte Carlo method, choosing the temperature and chemical potential difference $\Delta \mu$ between the two species as the given independent variables. Varying for $\Delta \mu=0$ the temperature and particle number $N$ over a wide range, the location of the coexistence curve in the thermodynamic limit is estimated. Well-equilibrated configurations from these Monte Carlo runs are used as initial states for microcanonical Molecular Dynamics runs, in order to study the microscopic structure and the behavior of transport coefficients as well as dynamic correlation functions along the coexistence curve. Dynamic structure factors $S_{\alpha \beta}(q, t)$ (and the corresponding static functions $\left.S_{\alpha \beta}(q)\right)$ are recorded $(\alpha, \beta, \in \mathrm{A}, \mathrm{B}), q$ being the wavenumber and $t$ the time, as well as the mean square displacements of the particles (to obtain the self-diffusion constants $D_{\mathrm{A}}, D_{\mathrm{B}}$ ) and transport coefficients describing collective transport, such as the interdiffusion constant and the shear viscosity. The minority species is found to diffuse a bit faster than the majority species. Despite the presence of strong concentration fluctuations in the system the Stokes-Einstein relation is a reasonable approximation.
\end{abstract}




\section{Introduction}

While the interplay between static structure and dynamical properties of simple fluids is a problem intensively studied both by analytic theory [1,2, 3, 4, 5] and simulations [5, 6, 7, 8, 10, and also extensions to rather complex fluids such as polymers are already given $91011 \quad 121314$, somewhat less attention has been paid to simple liquid mixtures: while static properties of liquid-liquid miscibility have been analyzed in some detail both by analytic theory (see e.g. [15]) and

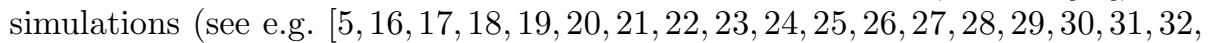
33 34 35, 36 ), dynamic properties of fluid mixtures have been investigated by computer simulation only occasionally (e.g. 5, 35, 37, 38, 39, 40,41,42]). In most of these studies, the precise phase behavior of the used model is not known, and also very small system sizes were used, and hence the proper interpretation of the results is difficult.

The present work aims to contribute towards filling this gap by studying both phase behavior and static structure in conjunction with dynamic correlations and transport phenomena. We address the simplest possible case, a symmetric binary Lennard-Jones mixture with a miscibility gap. Extensions of our approach to asymmetric Lennard-Jones mixtures and models suitable for a realistic description of binary mixtures of liquid metals (such as 35]) will be given in future work. We note that a specific asymmetric binary Lennard-Jones mixture with $80 \%$ A and $20 \%$ B particles is a very popular model for the study of the dynamics of glass-forming fluids [43, 44, 45]. However, the static phase diagram of that model system is not yet known.

The investigation of a symmetric mixture is of particular interest, because one can clearly distinguish in this case long-ranged correlations in the vicinity of the critical point from the different local ordering of A- and B- particles that drives the unmixing transition well below the critical temperature $T_{\mathrm{c}}$ : At the critical point a mixture of $50 \% \mathrm{~A}$ - and $50 \%$ B-particles is formed, i.e. $x_{\mathrm{B}}=$ $1-x_{\mathrm{A}}=N_{\mathrm{B}} /\left(N_{\mathrm{A}}+N_{\mathrm{B}}\right)=0.5\left(N_{\mathrm{A}}\right.$ and $N_{\mathrm{B}}$ being the number of $\mathrm{A}$ and $\mathrm{B}$ particles in the simulation box, respectively), and due to the symmetry of the model static A-A and B-B correlations are identical in the latter case. Well below $T_{\mathrm{c}}$ at coexistence one of the species, say $\mathrm{B}$, is the minority species and a $\mathrm{B}$ particle prefers a larger number of neighboring $\mathrm{B}$ particles than in the onephase region at the same concentration of B particles. We shall discuss below how this interplay of long-ranged correlations in the vicinity of $T_{\mathrm{c}}$ and local ordering far below $T_{\mathrm{c}}$ along the coexistence states affects the dynamics of the mixture.

For a symmetric mixture the phase diagram and other static properties are most easily computed with Monte Carlo (MC) methods using the semigrandcanonical ensemble rather than Molecular Dynamics (MD) methods. This type of MC method has been used with great success to establish binary alloy phase diagrams in the solid phase, both for lattice 46, 47, 48, 49] and off-lattice [50] models. It was also used to study lattice models of polymers [16 25, 27, 29],

and, in conjunction with finite size scaling methods, to investigate the critical behavior of symmetric binary Lennard-Jones fluids [32]. It is also suit- 
able for asymmetric models of fluid mixtures [17,35]. In this work we combine semi-grandcanonical MC simulations with microcanonical MD runs (i.e. at constant energy $E$ and constant volume $V$ ). The former simulations yield wellequilibrated configurations at concentrations $x_{\mathrm{B}}=1-x_{\mathrm{A}}$ and temperatures $T$ along the two-phase coexistence curve of the system and thus, these configurations can be used as initial states for the MD to study the structure and dynamics at coexistence and also in the vicinity of coexistence by changing the temperature or the composition.

The rest of the Paper is organized as follows. In the next section we shall briefly comment on the model and the used simulation techniques. A precise definition of the quantities that are computed is given in Sec. 3. Then we present the results for the structure (Sec. 4) and dynamics (Sec. 5) of our model that have been obtained from MD runs. Finally, Sec. 5 presents our conclusions and gives an outlook on further work.

\section{Model and simulation methods}

We consider a binary mixture of point-like particles interacting with LennardJones potentials $(\alpha, \beta \in \mathrm{A}, \mathrm{B})$,

$$
u\left(r_{i j}\right)=4 \varepsilon_{\alpha \beta}\left[\left(\frac{\sigma_{\alpha \beta}}{r_{i j}}\right)^{12}-\left(\frac{\sigma_{\alpha \beta}}{r_{i j}}\right)^{6}\right], \quad r_{i j}=\left|\vec{r}_{i}-\vec{r}_{j}\right|,
$$

where $\left\{\vec{r}_{i}\right\}$ are the positions of the particles, and the Lennard-Jones parameters $\varepsilon_{\alpha \beta}$ are chosen as follows

$$
\sigma_{\mathrm{AA}}=\sigma_{\mathrm{BB}}=\sigma_{\mathrm{AB}}=\sigma, \quad \varepsilon_{\mathrm{AA}}=\varepsilon_{\mathrm{BB}}=\varepsilon, \quad \delta \equiv \varepsilon_{\mathrm{AB}} / \varepsilon .
$$

We measure all lengths in units of $\sigma \equiv 1$, and we also choose Boltzmann's constant $k_{B} \equiv 1$. The temperature $T$ will be measured in units of $\varepsilon \equiv 1$, and (in the MD part) the masses of the two particle species are also chosen the same, $m_{\mathrm{A}}=m_{\mathrm{B}}=1$. The potential is truncated and shifted at $r_{i j}=2.5 \sigma$.

The same model has been studied earlier by Wilding [32] (note that in this work the potential was also truncated at $r_{i j}=2.5 \sigma$ but not shifted, and thus minor differences in the properties between our model and that of Ref. [32 must be expected). In the latter work, also the density of the binary fluid was varied over a wide range, $0 \leq \rho \leq 0.7$, and it was found for $\delta=0.7$, that the $\lambda$-line of critical points for fluid-fluid phase separation ends at a critical end point at a density of about $\rho \sigma^{3} \approx 0.6$ at the coexistence curve of liquid-gas phase separation. At sufficiently smaller values of $\delta$ than 0.7 the $\lambda$ line moves towards the liquid-gas critical point where it merges into a tricritical point and one may expect that this happens at densities below $\rho \sigma^{3} \approx 0.632$. In the present work, we are not interested in the liquid-gas transition of the model at all, and hence work at a much higher density, namely $\rho=1.0$. For this density we stay in the fluid phase for the whole temperature range of interest, i.e. $1 \leq T \leq 1.8$. 
Choosing $\delta=0.5$, the critical temperature of unmixing is $T_{c} \approx 1.638 \pm 0.005$ at this density, see Fig. 2 below.

The equilibration of the model is done as follows. We start from random configurations with an equal number of $\mathrm{A}$ and $\mathrm{B}$ particles. The particles are then displaced by means of a standard Monte Carlo scheme in the NVT ensemble, i.e. at constant total particle number $N$, constant volume $V$, and constant temperature $T$, rejecting or accepting a trial move according to the standard Metropolis criterion. Thereby, the length of a trial displacement is a randomly chosen number in the interval $[-\sigma / 20, \sigma / 20]$. After $100000 \mathrm{MC}$ steps the semigrandcanonical $\mathrm{MC}$ is switched on: At the end of each displacement step an attempted identity switch $(\mathrm{B} \longrightarrow \mathrm{A}$ or $\mathrm{A} \longrightarrow \mathrm{B})$ of $N / 10$ randomly chosen particles is made, which is again accepted or rejected according to a standard Metropolis criterion, where both the energy change $\Delta E$ and the change of the chemical potential $\pm \Delta \mu$ needs to be taken into account in the Boltzmann factor 7, 9, 10,50,51. Each configuration after this Monte Carlo move (whether the change was accepted or not) is counted as a state for the Monte Carlo averaging in the semi-grandcanonical ensemble, which is defined by the independent variables $N, V, T$, and the chemical potential differences $\Delta \mu$. Note that the relative concentration $x_{\mathrm{B}}=N_{\mathrm{B}} /\left(N_{\mathrm{A}}+N_{\mathrm{B}}\right)=N_{\mathrm{B}} / N$ is a fluctuating quantity and its average value is an output rather than an input to the simulation. Only for the choice $\Delta \mu=0$ the symmetry of the model between $\mathrm{A}$ and $\mathrm{B}$ dictates that for $T \geq T_{c}$ we must have $\left\langle x_{\mathrm{B}}\right\rangle=1 / 2$, while for $T<T_{c}$ we obtain for $\Delta \mu=0$ states at the two-phase coexistence curve. This is illustrated in Fig. 1] where the concentration distribution function $P\left(x_{\mathrm{A}}\right)$ is plotted for three temperatures. Note that we have done 5 independent runs, each with a length of $400000 \mathrm{MC}$ steps in the semi-grandcanonical ensemble, to calculate the distribution functions in Fig. [1 whereby we started the averaging after $100000 \mathrm{MC}$ steps in each run. In principle, for a finite system there is no symmetry breaking possible in full thermal equilibrium, and hence one should observe always two peaks, one at $x_{\mathrm{A}}^{\operatorname{coex}(2)}$ and the other one at $x_{\mathrm{A}}^{\operatorname{coex}(1)}=1-x_{\mathrm{A}}^{\operatorname{coex}(2)}$, corresponding to the two branches of the two-phase coexistence curve. In practice, these two branches are separated at low temperatures by a huge free energy barrier in phase space. Therefore, as can be seen in Fig. 10 at $T=1.2$ and $T=1.4$ only the A-rich phase is observed, and no transition to the B-rich side of the coexistence curve has

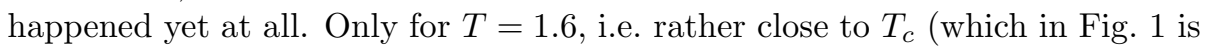
evident from the large width of the peaks), transitions back and forth between the two coexisting phases have occurred (although there were not enough of those transitions to make $P\left(x_{\mathrm{A}}\right)$ strictly symmetric around $x_{\mathrm{A}}^{\text {crit }}=1 / 2$ ).

This "ergodicity breaking" due to finite observation time in the context of first-order phase transitions is a well-known phenomenon [51] and does not hamper our analysis at all. From data such as shown in Fig. 10 wence obtain, when we normalize the part of $P\left(x_{\mathrm{A}}\right)$ for $x_{\mathrm{A}}>1 / 2$ to $\int P\left(x_{\mathrm{A}}\right) d x_{\mathrm{A}}=1$, both estimates for the location of the coexistence curve and the "concentration susceptibility" 
$\chi$,

$$
x_{\mathrm{A}}^{\operatorname{coex}(2)}=\int_{1 / 2}^{1} x_{\mathrm{A}} P\left(x_{\mathrm{A}}\right) d x_{\mathrm{A}} \quad, \quad k_{B} T \chi=N\left(\int_{1 / 2}^{1} x_{\mathrm{A}}^{2} P\left(x_{\mathrm{A}}\right) d x_{\mathrm{A}}-\left[x_{\mathrm{A}}^{\operatorname{coex}(2)}\right]^{2}\right) .
$$

In order to investigate the dynamics of well-equilibrated states precisely at the coexistence curve, we store a number of $n=10$ independent configurations at each of the 5 independent runs which have a number $N_{\mathrm{A}}$ of A particles such that the resulting ratio $x_{\mathrm{A}}$ is as close to $x_{\mathrm{A}}^{\operatorname{coex}(2)}$ as possible (note that $x_{\mathrm{A}}$ is "quantized", because $N_{A}, N$ are integers, but for large $N$ the discreteness of $x_{A}$, which is $1 / N$, is smaller than the statistical error with which $x_{\mathrm{A}}^{\operatorname{coex}(2)}$ can be estimated, and therefore this effect does not matter). With these configurations we can realize microcanonical MD runs right at the coexistence curve. Note that we have used in this work a standard MD code applying the velocity Verlet algorithm with a time step $\delta t=0.01$ in units of the time $t_{0}=\left(m \sigma^{2} / 48 \varepsilon\right)^{1 / 2}$ \}.

When we work in the one phase region we just heat up the configurations that we have gotten from the semi-grandcanonical Monte-Carlo at coexistence and we equilibrate the system by MD at the desired temperature $T_{\mathrm{f}}$. The temperature is then kept constant by coupling the system to a stochastic heat bath, i.e. every $50 \mathrm{MD}$ steps the velocities are replaced by new ones that are chosen randomly from a Boltzmann distribution corresponding to the temperature $T_{\mathrm{f}}$.

We have described these simple and almost straightforward considerations $\underline{54}$ in such detail here, because the literature is still full of examples where the authors are not aware of the pitfalls presented to equilibration by the presence of slowly relaxing variables. In the present problem, the conservation of the concentration (in the ensemble where both $N_{\mathrm{A}}$ and $N_{\mathrm{B}}$ are held fixed) together with the presence of a large correlation length of concentration fluctuations (which for $N \rightarrow \infty$ diverges at the critical point and causes the critical slowing down [53]) may lead to a very slow relaxation. Hydrodynamic slowing down is eliminated by the choice of an ensemble where the slow variable is not conserved, in our case the semi-grandcanonical ensemble. Critical slowing down cannot be avoided (apart from the special case of Ising models on lattices, for which cluster algorithms have been put forward [55]56] that eliminate also critical slowing down to a large extent). In the present work, we hence do not attempt to study the immediate vicinity of the critical point.

Fig. 2] shows the coexistence curve as estimated from our semi-grandcanonical method. One can see clearly that for $T \leq 1.4$ finite size effects on the coexistence curve are quite negligible, if $N \geq 400$. For $T=1.5$, data for $N=400$ overestimate the width of the two phase region very slightly, while data for $N=$ 800,1600 and 3200 agree within statistical errors. For $T=1.6$, however, data for both $N=400$ and $N=800$ overestimate the width of the coexistence curve slightly, while the data for both $N=1600$ and $N=3200$ still agree. For this reason, we have chosen $N=1600$ as the standard particle number, for which all analysis of static and dynamic correlations have been made (Secs. 3, 4). We 
have estimated the critical temperature $T_{c}$ from power law fits of the form

$$
f\left(x_{\mathrm{B}}\right)=1 / 2 \pm x_{\mathrm{B}}^{\text {coex }}=\widehat{B}\left(1-T / T_{c}\right)^{\beta}
$$

with the critical exponent $\beta=0.33$ which corresponds to the universality class of the three-dimensional Ising model. $\widehat{B}$ and $T_{c}$ have been used as adjustable parameters. For $N=400$ the fit yields $T_{c} \approx 1.666 \pm 0.005$, while for all larger values of $N$ the result is $T_{c} \approx 1.638 \pm 0.005$ (see Fig. 22). We emphasize at this point, however, that we deliberately do not address the problem of analyzing the critical behavior of transport coefficients in our study: for such a purpose one would need to work with system sizes $N$ that are several orders of magnitude larger, and extremely accurate data in the temperature range $1.6 \leq T \leq 1.7$ would be needed. This interesting problem would require substantially more computer resources than were available to us. In addition, we would have to locate $T_{c}$ much more accurately with a finite size scaling analysis $9,16,25,27$, [29,32,50,51]. Even though we do not study critical behavior, our choice of $N$ is much larger than most of the choices made in previous work on the dynamics of mixtures (e.g. Vogelsang and Hoheisel [39] work with $N=108$ and $N=256$ particles, and most data of Asta et al. 35] refer to $N=500$ particles).

\section{$3 \quad$ Static and dynamic quantities}

We now proceed to define the quantities that are computed in our simulations. With respect to static quantities, we have monitored the standard pairwise radial distribution functions $g_{\alpha \beta}(r)$ between the different pairs $\{\alpha, \beta \in(\mathrm{A}, \mathrm{B})\}$ of particles [1, $2,3,4,5,6,7,8$,

$$
g_{\alpha \beta}(r)=\frac{N}{\rho N_{\alpha} N_{\beta}}\left\langle\sum_{i=1}^{N_{\alpha}} \sum_{j=1}^{N_{\beta}} \delta\left(r-\left|\vec{r}_{i}(0)-\vec{r}_{j}(t)\right|\right)\right\rangle .
$$

The prime in the second sum means that $i=j$ has to be left out if $\alpha=\beta$.

In terms of the $g_{\alpha \beta}(r)$, partial structure functions $S_{\alpha \beta}(q)$ are defined as follows [2]:

$$
S_{\alpha \beta}(q)=x_{\alpha} \delta_{\alpha \beta}+x_{\alpha} x_{\beta} \rho \int_{0}^{\infty} g_{\alpha \beta}(r) \frac{\sin (q r)}{q r} 4 \pi r^{2} d r .
$$

with $x_{\alpha}=N_{\alpha} / N(\alpha \in \mathrm{A}, \mathrm{B})$. In order to compute the partial structure factors we used the formula

$$
S_{\alpha \beta}(\vec{q})=\frac{1}{N} \sum_{k=1}^{N_{\alpha}} \sum_{l=1}^{N_{\beta}}\left\langle\exp \left(i \vec{q} \cdot \vec{r}_{k l}\right)\right\rangle
$$

and performed the angular integration numerically to obtain $S_{\alpha \beta}(q)$. 
It turns out that it is more useful to form the following linear combinations of the radial distribution functions $g_{\alpha \beta}$ and the partial structure factors $S_{\alpha \beta}$ which describe correlations in number density $\left(g_{n n}\right.$ and $\left.S_{n n}\right)$, concentration correlations $\left(g_{c c}\right.$ and $\left.S_{c c}\right)$, and cross correlations between number density and concentration $\left(g_{n c}\right.$ and $\left.S_{n c}\right)$ [2,57,58. The real space functions are given by

$$
\begin{aligned}
g_{n n}(r) & =x_{\mathrm{A}}^{2} g_{\mathrm{AA}}(r)+2 x_{\mathrm{A}} x_{\mathrm{B}} g_{\mathrm{AB}}(q)+x_{\mathrm{B}}^{2} g_{\mathrm{BB}}(r), \\
g_{c c}(r) & =x_{\mathrm{A}}^{2} x_{\mathrm{B}}^{2}\left[g_{\mathrm{AA}}(r)+g_{\mathrm{BB}}(r)-2 g_{\mathrm{AB}}(r)\right], \\
g_{n c}(r) & =x_{\mathrm{A}} x_{\mathrm{B}}\left[x_{\mathrm{A}} g_{\mathrm{AA}}(r)-x_{\mathrm{B}} g_{\mathrm{BB}}(r)+\left(x_{\mathrm{B}}-x_{\mathrm{A}}\right) g_{\mathrm{AB}}(r)\right],
\end{aligned}
$$

and the functions in reciprocal space by

$$
\begin{aligned}
S_{n n}(q) & =S_{\mathrm{AA}}(q)+2 S_{\mathrm{AB}}(q)+S_{\mathrm{BB}}(q), \\
S_{c c}(q) & =x_{\mathrm{B}}^{2} S_{\mathrm{AA}}(q)+x_{\mathrm{A}}^{2} S_{\mathrm{BB}}(q)-2 x_{\mathrm{A}} x_{\mathrm{B}} S_{\mathrm{AB}}(q), \\
S_{n c}(q) & =x_{\mathrm{B}} S_{\mathrm{AA}}(q)-x_{\mathrm{A}} S_{\mathrm{BB}}(q)+\left(x_{\mathrm{B}}-x_{\mathrm{A}}\right) S_{\mathrm{AB}}(q) .
\end{aligned}
$$

We will see below that strong concentration fluctuations are observed if one approaches the critical point of unmixing (and this happens not only in the immediate vicinity of the critical point). One can try to describe $S_{c c}(q)$ at small $q$ by the Ornstein-Zernike form,

$$
S_{c c}(q)=\frac{k_{B} T \chi}{1+\xi^{2} q^{2}}
$$

where the "susceptibility" $\chi$ is given by Eq. (3) and $\xi$ has the meaning of a static correlation length.

Turning to dynamic quantities, the most straightforward quantity to consider is the mean square displacement of tagged particles

$$
g_{\mathrm{A}}(t)=\left\langle\left[\vec{r}_{i, \mathrm{~A}}(0)-\vec{r}_{i, \mathrm{~A}}(t)\right]^{2}\right\rangle, \quad g_{\mathrm{B}}(t)=\left\langle\left[\vec{r}_{j, \mathrm{~B}}(0)-\vec{r}_{j, \mathrm{~B}}(t)\right]^{2}\right\rangle \quad,
$$

where it is understood that the average $\langle\cdots\rangle$ includes an average over all particles of type A or B, respectively. Another quantity which also monitors the motion of individual particles are the incoherent intermediate structure functions $F_{s}^{(\mathrm{A})}(q, t)$ and $F_{s}^{(\mathrm{B})}(q, t)$,

$$
\begin{aligned}
& F_{s}^{(\mathrm{A})}(q, t)=\frac{1}{N_{\mathrm{A}}} \sum_{i \in \mathrm{A}}\left\langle\exp \left\{-i \vec{q} \cdot\left[\vec{r}_{i}(0)-\vec{r}_{i}(t)\right]\right\}\right\rangle, \\
& F_{s}^{(\mathrm{B})}(q, t)=\frac{1}{N_{\mathrm{B}}} \sum_{j \in \mathrm{B}}\left\langle\exp \left\{-i \vec{q} \cdot\left[\vec{r}_{j}(0)-\vec{r}_{j}(t)\right]\right\}\right\rangle,
\end{aligned}
$$

where the sum runs over all A particles \{Eq. (16) $\}$ or B particles \{Eq. (17) $\}$, respectively. Note that due to the isotropy of the fluid, Eqs. (16), (17) cannot depend on the direction of the scattering vector $\vec{q}$. When the displacements of the particles are Gaussian distributed, Eqs. (16), (17) reduce to

$$
F_{s}^{(\mathrm{A})}(q, t)=\exp \left[-\frac{1}{6} q^{2} g_{\mathrm{A}}(t)\right], \quad F_{s}^{(\mathrm{B})}(q, t)=\exp \left[-\frac{1}{6} q^{2} g_{\mathrm{B}}(t)\right] .
$$


Noting further the Einstein relations

$$
g_{\mathrm{A}}(t)=6 D_{\mathrm{A}} t, \quad g_{\mathrm{B}}(t)=6 D_{\mathrm{B}} t, \quad t \rightarrow \infty,
$$

where $D_{\mathrm{A}}$ and $D_{\mathrm{B}}$ are self-diffusion constants of the particles, we expect that the asymptotic decay of $F_{s}^{(\mathrm{A})}(q, t)$ and $F_{s}^{(\mathrm{B})}(q, t)$ can be described by a simple exponential variation with time,

$$
F_{s}^{(\mathrm{A})}(q, t) \propto \exp \left[-t / \tau_{\mathrm{A}}(q)\right], \quad F_{s}^{(\mathrm{B})}(q, t) \propto \exp \left[-t / \tau_{\mathrm{B}}(q)\right],
$$

and Eqs. (18)-(20) then imply

$$
\lim _{q \rightarrow 0}\left[\tau_{\mathrm{A}}(q) q^{2}\right]^{-1}=D_{\mathrm{A}}, \quad \lim _{q \rightarrow 0}\left[\tau_{\mathrm{B}}(q) q^{2}\right]^{-1}=D_{\mathrm{B}} .
$$

In addition, we introduce partial coherent intermediate structure functions $S_{\alpha \beta}(q, t)$ defined as $[\alpha, \beta \in(\mathrm{A}, \mathrm{B})]$

$$
S_{\alpha \beta}(q, t)=\frac{1}{N} \sum_{\substack{i=1 \\ i \in \alpha}}^{N_{\alpha}} \sum_{\substack{j=1 \\ j \in \beta}}^{N_{\beta}}\left\langle\exp \left\{-i \vec{q} \cdot\left[\vec{r}_{i}(0)-\vec{r}_{j}(t)\right]\right\}\right\rangle,
$$

and from these functions we can define number density and concentration correlation as well as cross correlation functions $\left\{S_{n n}(q, t), S_{c c}(q, t)\right.$ and $\left.S_{n c}(q, t)\right\}$ by forming exactly the same type of linear combinations as written in Eqs. (11)(13), but with the $S_{\alpha \beta}(q, t)$ rather than their static counterpart $S_{\alpha \beta}(q)$. In order to compare the time correlation functions $S_{\alpha \beta}(q, t)$ for different values of $q$ it is convenient to consider the normalized functions

$$
F_{\alpha \beta}(q)=\frac{S_{\alpha \beta}(q, t)}{S_{\alpha \beta}(q)} .
$$

The normalized functions $F_{n n}(q, t), F_{c c}(q, t)$, and $F_{n c}(q, t)$ are defined in a similar way by normalizing $S_{n n}(q, t), S_{c c}(q, t)$ and $S_{n c}(q, t)$ by the corresponding static correlation function.

Further quantities of interest are the instantaneous values of the components of the pressure tensor $\left(x, y, z\right.$ denote the Cartesian components, $\vec{v}_{i}$ is the velocity of the $i$ 'th particle)

$$
\sigma_{x y}(t)=\sum_{i=1}^{N}\left[m_{i} v_{i x} v_{i y}+\frac{1}{2} \sum_{j(\neq i)}\left|x_{i}-x_{j}\right| F_{y}\left(\left|\vec{r}_{i}-\vec{r}_{j}\right|\right)\right],
$$

$\vec{F}$ being the force acting between particles $i, j$. From the autocorrelation function of $\sigma_{x y}(t)$ one gets the shear viscosity $\eta$ of the fluid by a Green-Kubo formula [1,2],

$$
\eta=\frac{1}{V k_{B} T} \int_{0}^{\infty} d t\left\langle\sigma_{x y}(0) \sigma_{x y}(t)\right\rangle
$$


Finally we mention the Green-Kubo formula for the interdiffusion constant (note that we have chosen the masses of all particles equal to each other here) [2]

$$
D_{\mathrm{int}}=\frac{1}{N S_{c c}(0)} \int_{0}^{\infty} d t\left\langle J_{x}^{\mathrm{int}}(0) J_{x}^{\mathrm{int}}(t)\right\rangle,
$$

where the current $\vec{J}^{\text {int }}(t)$ is defined as follows:

$$
\vec{J}^{\mathrm{nt}}(t)=x_{\mathrm{B}} \sum_{i=1}^{N_{\mathrm{A}}} \vec{v}_{i}(t)-x_{\mathrm{A}} \sum_{i=1}^{N_{\mathrm{B}}} \vec{v}_{i}(t)
$$

One can easily calculate $D_{\text {int }}$ for an ideal mixture [such a mixture is formed if the particles are labeled but are otherwise identical and thus the entropy of mixing is equal to $\left.-N k_{B}\left(x_{\mathrm{A}} \log x_{\mathrm{A}}+x_{\mathrm{B}} \log x_{\mathrm{B}}\right)\right]$. In this case cross correlations in the autocorrelation function for $J_{x}^{\text {int }}$ in Eq. (26) vanish and the interdiffusion constant can be expressed by the self-diffusion constants [2,

$$
D_{\text {int }}=x_{\mathrm{B}} D_{\mathrm{A}}+x_{\mathrm{A}} D_{\mathrm{B}} .
$$

It has been shown by MD simulations that Eq. (28) is a good approximation for mixtures of Lennard-Jones fluids in the one-phase region [38, 59. However, for ideal mixtures on a rigid lattice (where $\mathrm{A}$, B-atoms hop with jump rates $\sigma_{\mathrm{A}}, \sigma_{\mathrm{B}}$ to vacant sites, assuming that a small number of vacancies is present) Eq. (28) does not hold [60.

\section{Static properties of the symmetrical binary fluid mixture}

In this section we analyze the structure of the symmetric Lennard-Jones mixture. Results are shown along the coexistence line and along the different paths in the one-phase region that are indicated in Fig. 2]

In Fig. B we present the pair correlation functions $g_{\alpha \beta}(r)$ for the three states at temperatures $T=1.2,1.4$, and 1.6 at the coexistence curve (cf. Fig. 21). These data show a typical normal fluid behavior in all cases, as expected. Although it would be hard to recognize from these partial radial distribution functions that one approaches the critical point of unmixing in a binary fluid, the comparison of the different correlations shows nontrivial features. At each temperature, the first peak in $g_{\mathrm{AB}}(r)$ is at a slightly smaller distance than in $g_{\mathrm{AA}}(r)$ and $g_{\mathrm{BB}}(r)$. Moreover, the second peak in $g_{\mathrm{BB}}(r)$ is shifted towards smaller distances compared to the other two functions and this effect is more pronounced the lower the temperature is, i.e. the farther one is away from the critical point. The explanation of this feature is simple: The $\mathrm{B}$ particles are the minority species at the considered state points and thus, at small concentrations $x_{\mathrm{B}}$ it is very likely that one finds an A particle between two next-nearest B particles forming 
a B-A-B sequence. Since the distance between nearest A-B neighbors is smaller than that between nearest $\mathrm{A}-\mathrm{A}$ and $\mathrm{B}-\mathrm{B}$ neighbors one may expect that a large amount of such B-A-B sequences, where the distance between next-nearest $\mathrm{B}$ particles is smaller than in a B-B-B sequence, leads to the shift in the second peak of $g_{\mathrm{BB}}(r)$. Certainly, the difference between $g_{\mathrm{AA}}$ and $g_{\mathrm{BB}}$ becomes less pronounced the closer one approaches $x_{\mathrm{B}}=0.5$ (and thus the critical point) where, due to the symmetry of our model, $g_{\mathrm{AA}}$ and $g_{\mathrm{BB}}$ are identical.

The temperature dependence of $g_{\mathrm{AA}}(r)$ and $g_{\mathrm{BB}}(r)$ in the one-phase region is shown in Fig. 团 for the constant concentration $x_{\mathrm{B}}=0.10375$ in the temperature range $1.4 \leq T \leq 1.8$ ( $T=1.4$ corresponds to a state on the coexistence curve). We see that the temperature dependence is very weak for $g_{\mathrm{AA}}(r)$ whereas we observe a significant increase of the amplitude of the first peak in $g_{\mathrm{BB}}(r)$ decreasing the temperature towards the coexistence line. The latter effect is even more pronounced in the coordination number function $z_{\alpha \alpha}(r)$ that is shown in the insets of Fig. 团 This function is defined by

$$
z_{\alpha \alpha}(r)=x_{\alpha} \int_{0}^{r} d r^{\prime} 4 \pi r^{\prime 2} g_{\alpha \alpha}\left(r^{\prime}\right) \quad \alpha \in[\mathrm{A}, \mathrm{B}]
$$

which gives the number of particles of type $\alpha$ surrounding a particle of type $\alpha$ within a distance $r^{\prime}<r$. In the considered temperature range $z_{\mathrm{BB}}(r)$ changes at $r=1.4$ (i.e. around the location of the first minimum) from $z_{\mathrm{BB}}=1.37$ for $T=1.8$ to $z_{\mathrm{BB}}=1.55$ for $T=1.4$. By a closer inspection of $z_{\mathrm{AA}}(r)$ one finds that the changes in this quantity are of the same order. Thus there are only minor changes in the local order if one approaches the coexistence line from above.

We now proceed to discuss the behavior of the structure factors $S_{n n}(q)$, $S_{c c}(q)$, and $S_{n c}(q)$ \{Eqs. (11)-(13) $\}$ along the coexistence line and for the latter two quantities also the corresponding functions in real space $\{$ Eqs. (9)-(10) $\}$, see Fig. .5. The number density structure factor $S_{n n}(q)$ behaves exactly as expected for any dense simple liquid, and in particular $S_{n n}(q \rightarrow 0)$ is very small, as expected for liquids that are almost incompressible. The height of the first peak is around 3 , as is typical for fluids at temperatures somewhat higher than the melting temperature, and there is a pronounced second peak, due to the rather regular close packing of atoms in a dense fluid.

In contrast, $S_{c c}(q)$ at $T=1.2$ is very small and structureless throughout: in symmetrical binary mixtures, there is very little coupling between density and concentration fluctuations. For $T=1.4$, we see already some enhancement of $S_{c c}(q)$ at small $q$, reflecting the growing concentration fluctuations. For $T=1.6$, at small $q$ a rather dramatic growth of $S_{c c}(q \rightarrow 0)$ signals the proximity of the critical point. For small $q$ we have fitted the data by the Ornstein-Zernike form Eq. (14) (bold lines in Fig. 5b), where we have used the correlation length $\xi$ as an adjustable parameter and we have estimated the susceptibility $\chi$ by means of Eq. (31) from the distribution function $P\left(x_{\mathrm{A}}\right)$ (the values of $S_{c c}(0)=k_{B} T \chi$ are shown as crosses in Fig. 5 ). From the fits we recognize that the data for small $q$ can be described by Eq. (14) within the statistical errors (note that there is only one adjustable parameter, the correlation length $\xi$ ). From the fits we find 
a growing correlation length $\xi$ with increasing temperature towards $T_{c}$. This behavior can be also inferred from $g_{c c}(r)$ (see inset of Fig. [5) at $T=1.6$ which has a rather pronounced tail for $r>2$. Also remarkable in $g_{c c}(r)$ is the negative peak around $r \approx 0.9$ which stems from the fact that these distances are avoided by nearest A-A and B-B neighbors but are typical for nearest A-B neighbors.

Particularly interesting is the structure factor $S_{n c}(q)$. In a mixture with $\Delta \mu=0$ at $T>T_{c}$, where then $x_{\mathrm{A}}=x_{\mathrm{B}}=1 / 2$, we expect that $S_{n c}(q) \equiv 0$ due to the perfect symmetry between $\mathrm{A}$ and $\mathrm{B}$. Along the coexistence curve, the spontaneous symmetry breaking between A and B invalidates this argument, and hence a nonzero $S_{n c}(q)$ is possible. As we see in Fig. 5t this is obviously the case. The origin of the oscillations in $S_{n c}(q)$ can be most easily understood by means of its counterpart in real space, $g_{n c}(r)$ (see inset of Fig. 5 ). The most pronounced feature in the latter quantity is a peak with negative intensity around $r_{1} \approx 0.9$. This feature is again due to the fact that the first peak in $g_{\mathrm{AB}}(r)$ is slightly shifted to smaller distances compared to the first peak in $g_{\mathrm{AA}}$ and in $g_{\mathrm{BB}}$ such that the intensity of the latter two functions in the interval $0.85<r<0.9$ is essentially zero whereas $g_{\mathrm{AB}}$ is rapidly increasing for the latter distances. This leads to the occurrence of negative correlations in $g_{n c}$ and thus to the peak around $r_{1}$. We emphasize that the scale for $S_{n c}(q)$ and $g_{n c}(r)$ is clearly very much smaller than for the other structure factors and pair correlation functions, respectively.

Similar data have been taken for two other paths in the phase diagram of Fig. 2. namely one at constant temperature, $T=1.5$, varying $\Delta \mu$ (and hence $x_{\mathrm{B}}$ ), and one at constant $x_{B}=0.10375$. It turns out that the radial distribution functions $g_{\alpha \beta}(r)$ always look similar to those that are shown in Fig. 3] and hence are not shown here. The same statement applies to $S_{n n}(q)$, which exhibits only a weak dependence on either temperature or composition. Here we hence show only those quantities which have a more pronounced dependence on our control parameters, namely $S_{c c}(q)$ and $S_{n c}(q)$, Figs. (6), (7). From Fig. [6 we see that the enhancement of $S_{c c}(q \rightarrow 0)$ persists to rather high temperatures, and also $S_{n c}(q)$ is only weakly temperature-dependent in the temperature region shown. In contrast, when we change the composition at $T=1.5$, we find that $S_{c c}(q)$ quickly loses all structure when $x_{\mathrm{B}} \rightarrow 0$ (Fig. $7 \mathrm{7}$ ), and a related trend is observed in $S_{n c}(q)$.

\section{Dynamic properties of the symmetrical binary fluid mixture}

The detailed description of the structure of our Lennard-Jones model that we have done so far is necessary to understand its dynamic properties. The next two sections are devoted to the results for time-dependent correlation functions and transport coefficients. In Sec. 5.1 we analyze the dynamic properties of our system along the fluid-fluid coexistence line whereas in Sec. 5.2 dynamic quantities are discussed that have been obtained along the path at $x_{\mathrm{B}}=0.10375$ 
which starts in the one-phase region and ends at the coexistence line at $T=1.4$ (see Fig. 2).

\subsection{The dynamics at phase coexistence}

We start by showing the incoherent intermediate structure function for the A particles, $F_{s}^{(\mathrm{A})}(q, t)\{$ Eq. 16) $\}$, see Fig. 8 (data for B-particles look very similar and therefore are not shown). Note that for $q \approx 7$, where the peaks of the static structure factors $S_{\alpha \beta}(q)$ and $S_{n n}(q)$ occur, $F_{s}^{\alpha}(q, t), \alpha \in(\mathrm{A}, \mathrm{B})$, decays on the time scale of about $t=20$ and this decay is certainly even faster for $q=10$. Moreover, the temperature dependence of the $F_{s}^{\alpha}(q, t)$ is weak in the temperature range under consideration. Such a short structural relaxation time as well as a weak temperature dependence indeed are expected for any ordinary fluid, which has no tendency to glass formation [43 44, 45].

On the other hand, for small $q$ the decay of $F_{s}^{(\mathrm{A})}(q, t), F_{s}^{(\mathrm{B})}(q, t)$ is much slower, and this slowing down simply reflects the diffusive behavior predicted in Eqs. (18)-(21). Fig. 9 shows that this interpretation in fact is nicely consistent with the data in that, in agreement with Eq. (30), the product $\tau_{\alpha} q^{2}$ approaches the inverse diffusion constant $D_{\alpha}$ for $q \rightarrow 0$ (the values for $D_{\alpha}^{-1}$ are indicated as arrows in Fig. 9). One can further see that the B particles diffuse a bit faster than the A particles. Of course, we expect that this dynamic asymmetry must vanish when we are at the critical point, and indeed, as one can infer from Fig. 9 the ratio $D_{\mathrm{B}} / D_{\mathrm{A}}$ decreases to one if the critical point is approached from below along the coexistence line. The difference in the diffusion constants has a simple reason: In the A rich phase the B particles find mainly A particles as nearest neighbors, and since the interaction between particles of different species is weaker than for equal particles it is easier for the B particles to escape from an $\mathrm{A}$ rich neighborhood than from a B rich one. Thus, the effective activation energy for the diffusion of $\mathrm{B}$ particles is smaller in an A rich than in an B rich environment. Note that the behavior of $\tau_{\alpha} q^{2}$ at finite $q$ is very different from liquids near a glass transition: In such liquids one would see a peak in $\tau_{\alpha} q^{2}$ around a $q$ value that corresponds to the location of the structure factor maximum (see Ref. 61] and references therein).

Particularly interesting is the behavior of the collective structure functions (Figs. 10 11). In $F_{\mathrm{AA}}(q, t)$ (Fig. 10) we observe again a very fast decay for $q$ values around the structure factor maximum $q=7$ and at $q=0.992$, i.e. at small $q$, the decay occurs on a two orders of magnitude larger time scale. For the latter value of $q$ one can clearly recognize that several relaxation processes contribute, since the decay emerges in two steps. Note that $F_{\mathrm{AB}}(q, t)$ and $F_{\mathrm{BB}}(q, t)$ exhibit a similar behavior and so are not shown here. In contrast to the partial structure functions, in the concentration-concentration correlation function $F_{c c}(q, t)$ only the slow decay is found at $q=0.992$ (Fig. 111). Surprisingly, this is also the case at $T=1.2$ although, at this temperature, there is almost no structure in the corresponding static function $S_{c c}(q)$. As can be also inferred from Fig. [1] the function $F_{n n}(q, t)$ shows a very fast decay to zero at $q=0.992$ (accompanied by 
oscillations due to acoustic modes), whereas it has a slightly slower relaxation to zero than $F_{c c}(q, t)$ at $q=7$, i.e. the location of the structure factor maximum.

The relaxation times for the collective correlation functions are of the same order as those detected from the self-correlation functions: disappointingly, there is no clear indication of critical slowing down when we approach $T_{c}$ from below, since in the temperature region studied the overall Arrhenius-like increase of the relaxation time as $T$ is lowered dominates (the Arrhenius-like behavior is demonstrated below in the case of the diffusion constants).

\subsection{The dynamics from the one phase region to coexis- tence}

The qualitative character of the relaxation functions $F_{s}^{(\mathrm{A})}(q, t), F_{s}^{(\mathrm{B})}(q, t)$ and $F_{\mathrm{AA}}(q, t), F_{c c}(q, t)$ in the one phase region is rather similar to the behavior found along the coexistence curve. Therefore, we do not show these functions in any detail here, but proceed immediately to a counterpart of Fig. [9 in Fig. 12 where now the relaxation times $\tau_{\mathrm{A}}(q) q^{2}$ and $\tau_{\mathrm{B}}(q) q^{2}$ extracted from the incoherent intermediate scattering functions at constant $x_{\mathrm{B}}=0.10375$ are shown as a function of wavenumber $q$. Again one finds that the minority species diffuses a bit faster than the majority. The reason for the larger discrepancies of $D_{\alpha}^{-1}$ and $\tau_{\alpha}(q) q^{2}$ for $q \rightarrow 0$ in the case of the B particles is of course due to the fact that the statistics is worse for the $\mathrm{B}$ particles which is the minority species.

This difference in the diffusion constants is emphasized in the Arrhenius plot, Fig. 13] where the logarithm of $D_{\alpha}$ is plotted vs. inverse temperature. It is seen that a simple thermally activated behavior (which would show up as straight lines in this plot) holds only over very restricted temperature regimes which might be related to the fact that there are small but significant changes in the local structure when approaching the coexistence line (see especially Fig. (4). Also plotted in Fig. 13 are the inverse shear viscosity $\eta^{-1}$ and the interdiffusion constant $D_{\mathrm{int}}$. For an accurate computation of these quantities we have undertaken a much larger effort than for the single particle quantitities: At each temperature we averaged over 50 independent runs of $400000 \mathrm{MD}$ steps to calculate $\eta$ and $D_{\text {int }}$ by means of Eq. (25) and Eq. (26), respectively. We show also in Fig. 13] the interdiffusion constant according to Eq. (28) that is valid for an ideal mixture. Already for $T<2.5 D_{\text {int }}$ decouples from the ideal mixture approximation and thus from the self-diffusion constants, and $D_{\text {int }}$ becomes significantly smaller than the $D_{\alpha}$ if one approaches the coexistence curve.

It is now interesting to check for the Stokes-Einstein relation, which relates to the self-diffusion constant of a diffusing spherical particle of diameter $d$ in a fluid of viscosity $\eta$ as

$$
D=\frac{k_{B} T}{2 \pi \eta d} .
$$

The factor 2 in the denominator of Eq. (30) corresponds to the assumption of slip boundary conditions on the surface of the diffusing particle (for stick boundary conditions a factor 3 instead of 2 appears) 62 . 
Using the data for $\eta, D_{\mathrm{A}}, D_{\mathrm{B}}$ and invoking Eq. (30) one obtains the corresponding effective Stokes-Einstein diameters $d_{\mathrm{A}}, d_{\mathrm{B}}$ shown in Fig. 14] The data for $d_{\alpha}$ show that despite the strong concentration fluctuations in the system the Stokes-Einstein relation is a good approximation. The finding that the values for $d_{\mathrm{A}}$ and $d_{\mathrm{B}}$ are different is reasonable since the distance for nearest $\mathrm{A}-\mathrm{B}$ neighbors is slightly smaller than that for A-A or B-B neighbors and the B particles as the minority species are mostly surrounded by A particles (see Fig. 4); thus, the effective hydrodynamic diameter for the B particles is slightly smaller than that for the A particles. Note that with the assumption of slip boundary conditions in Eq. (30) we obtain reasonable values around 1 for $d_{\mathrm{A}}$ and $d_{\mathrm{B}}$ whereas for stick boundary conditions these values are rather small (around 0.6).

\section{Concluding remarks}

The present paper considers as a simple model system for a binary fluid mixture with a miscibility gap a dense Lennard-Jones mixture with fully symmetrical interactions, $\sigma_{\mathrm{AA}}=\sigma_{\mathrm{BB}}=\sigma_{\mathrm{AB}}=\sigma, \varepsilon_{\mathrm{AA}}=\varepsilon_{\mathrm{BB}}=\varepsilon=1, \delta=\varepsilon_{A B}-\varepsilon=0.5$, and also the masses of the two types of particles are chosen the same, $m_{A}=m_{B}=m$. The aim of the present work was to carry out a feasibility study, where by combination of the semi-grandcanonical Monte Carlo technique with Molecular Dynamics simulations both static properties, including the phase diagram, and dynamic correlation functions and associated transport coefficients are obtained simultaneously. It is shown that outside of the critical region of the mixture rather small system sizes (such as a total number of $N=1600$ particles) already suffice to obtain quantitatively reliable results, while in the critical region (which could not yet be studied here) much larger sizes clearly are mandatory. Such large sizes would also be needed for the very interesting problem of spinodal decomposition of the binary fluid mixture after a quench from the one-phase region into the unstable region of the phase diagram (Fig. (2). Since our study is the first study of a fluid mixture where both the phase diagram and the relevant transport coefficients have been determined simultaneously, such an extension to far from equilibrium dynamics clearly would be very interesting.

Our studies of transport coefficients are distinct from earlier work on similar models by the fact the we know precisely where in the phase diagram the considered state points are for which transport coefficients have been determined. This fact clearly facilitates the proper interpretation of the results. A particular interesting finding concerns the asymmetric composition in our otherwise fully symmetric model.

In further work we also plan to study systematically Onsager coefficients to test the concepts of phenomenological irreversible thermodynamics for the present model and to check "mixing rules" for the interdiffusion coefficient. Moreover, we will present extensions to more realistic models of real materials which always exhibit asymmetry in the interactions, to allow also a comparison to suitable experiments. 
Acknowledgments: The present research was supported by the Deutsche Forschungsgemeinschaft (DFG) under grant $\mathrm{N}^{o}$ Bi314/18 (SPP 1120). One of the authors (J. H.) acknowledges the support of the DFG under grant $\mathrm{N}^{o} \mathrm{HO}$ 2231/2-1. 


\section{References}

[1] J.P. Boon and S. Yip, Molecular Hydrodynamics (McGraw Hill, New York, 1980).

[2] J.-P. Hansen and I.R. McDonald, Theory of Simple Liquids (Academic Press, London, 1986).

[3] J.P. Hansen, D. Levesque, and J. Zinn-Justin (eds.), Liquids, Freezing, and the Glass Transition (North-Holland, Amsterdam, 1991).

[4] U. Balucani and M. Zoppi, Dynamics of the Liquid State (Oxford Univ. Press, Oxford, 1994).

[5] C. Hoheisel, Theoretical Treatment of Liquids and Liquid Mixtures (Elsevier, Amsterdam, 1993).

[6] G. Ciccotti and G.G. Hoover (eds.), Molecular Dynamics Simulations of Statistical Mechanical System (North-Holland, Amsterdam, 1986).

[7] M.P. Allen and D.J. Tildesley, Computer Simulation of Liquids (Charendon Press, Oxford, 1987).

[8] D.C. Rapaport, The Art of Molecular Dynamics Simulation (Cambridge Univ. Press, Cambridge, 1995).

[9] K. Binder and G. Ciccotti (eds.), Monte Carlo and Molecular Dynamics of Condensed Matter Systems (Società Italiana di Fisica, Bologna, 1996).

[10] D. Frenkel and B. Smit, Understanding Molecular Simulation: From Algorithms to Applications (Academic Press, San Diego, 2002).

[11] K. Binder, in J. Bicerano (ed.), Computational Modelling of Polymers (M. Dekker, New York, 1992).

[12] M.P. Allen and D.J. Tildesley (eds.), Computer Simulations in Chemical Physics (Kluwer, Dordrecht, 1993).

[13] K. Binder (ed.), Monte Carlo and Molecular Dynamics Simulations in Polymer Science (Oxford Univ. Press, New York, 1995).

[14] M. Baus, L.F. Rull, and J.-P. Ryckaert (eds.), Observation, Prediction and Simulation of Phase Transitions in Complex Fluids (Kluwer, Dordrecht, 1995).

[15] J.S. Rowlinson and F.L. Swinton, Liquids and Liquid Mixtures (Butterworths, London, 1982).

[16] A. Sariban and K. Binder, J. Chem. Phys. 86, 5859 (1987).

[17] D.A. Kofke and E.D. Glandt, Mol. Phys. 64, 1105 (1988). 
[18] A.Z. Panagiotopolous, N. Quirke, M.R. Stapleton, and D.J. Tildesley, Mol. Phys. 63, 527 (1988).

[19] J.G. Amar, Mol. Phys. 4, 739 (1989).

[20] A.Z. Panagiotopolous, Int. J. Thermophys. 10, 447 (1989).

[21] M.E. van Leeuwen, C.J. Peter, J.D. Arons, and A.Z. Panagiotopolous, Fluid Phase Equilibria 66, 57 (1991).

[22] V.I. Harismiadis, N.K. Koutras, D.P. Tassios, and A.Z. Panagiotopoulos, Fluid Phase Equilibria 65, 1 (1991).

[23] R.D. Mountain and A.H. Harvey, J. Chem. Phys. 94, 2238 (1991).

[24] W.G.T. Kranendonk and D. Frenkel, Mol. Phys. 72, 699 (1991).

[25] H.-P. Deutsch and K. Binder, Macromolecules, 25, 6214 (1992).

[26] A.Z. Panagiotopoulos, Mol. Simul. 9, 1 (1992).

[27] M. Müller and K. Binder, Macromolecules 28, 1825 (1995).

[28] E. de Miguel, E. Martin del Rio, and M.M. Telo da Gama, J. Chem. Phys. 103, 6188 (1995).

[29] M. Müller and N.B. Wilding, Phys. Rev. E 51, 2079 (1995).

[30] N.B. Wilding, in D. Stauffer (ed.) Annual Reviews of Computational Physics IV, p. 37.

[31] N.B. Wilding, J. Phys.: Condens. Matter 8, 9637 (1996).

[32] N.B. Wilding, Phys. Rev. E 55, 6624 (1997).

[33] M.-O Ihm, F. Schneider, and P. Nielaba, Progr. Colloid. Polym. Sci. 104, 166 (1997).

[34] F.A. Escobedo, J. Chem. Phys. 110, 11999 (1999).

[35] M. Asta, D. Morgan, J.J. Hoyt, B. Sadigh, J.D. Althoff, D. de Fontaine, and S. M. Foiles, Phys. Rev. B 59, 1427 (1999).

[36] P. Virnau, M. Müller, L.G. MacDowell, and K. Binder, Comp. Phys. Comm. 147, 378 (2002).

[37] G. Jacucci and I.R. MacDonald, Physica 80A, 607 (1975).

[38] M. Schoen and C. Hoheisel, Mol. Phys. 52, 1029 (1984).

[39] R. Vogelsang and C. Hoheisel, Phys. Rev. A 98, 6296 (1988).

[40] H. Luo, C. Hoheisel and J. Karkheck, Phys. Rev. A 42, 4609 (1990). 
[41] H.P. van den Berg and C. Hoheisel, Phys. Rev. A 42, 3368 (1990).

[42] C. Hoheisel and G. Stell, Chem. Liq. 24, 63 (1991).

[43] W. Kob and H.C. Andersen, Phys. Rev. E 51, 4626 (1995); ibid 52, 4134 (1995).

[44] W. Kob, J. Phys.: Condens. Matter 11, R85 (1999).

[45] W. Kob, in Proc. Les Houches 2002 (to be publ.).

[46] K. Binder, Phys. Rev. Lett. 45, 811 (1980).

[47] K. Binder, J.L. Lebowitz, M.R. Phani, and M.H. Kalos, Acta Metall. 29, 1655 (1981).

[48] F. Schmid and K. Binder, J. Phys.: Condens. Matter 4, 3569 (1992).

[49] K. Binder, in Statics and Dynamics of Alloy Phase Transformations (P.E.A. Turchi and A. Ganis, Eds.), p. 467 (Plenum Press, New York, 1994).

[50] B. Dünweg and D.P. Landau, Phys. Rev. B 48, 14182 (1993).

[51] D.P. Landau and K. Binder, A Guide to Monte Carlo Simulations in Statistical Physics (Cambridge University Press, Cambridge, 2000).

[52] L.P. Kadanoff and P.C. Martin, Ann. Phys. (NY) 24, 419 (1963).

[53] P.C. Hohenberg and B.I. Halperin, Rev. Mod. Phys. 49, 435 (1977).

[54] K. Binder, in K. Binder (ed.) Monte Carlo Simulations in Statistical Physics, p. 1 (Springer, Berlin, 1979).

[55] R.H. Swendsen and J.S. Wang, Phys. Rev. Lett. 58, 861 (1987).

[56] U. Wolff, Phys. Rev. Lett. 62, 361 (1989).

[57] A.B. Bhatia and D.E. Thornton, Phys. Rev. B 52, 3004 (1970).

[58] J.M. Ziman, Models of disorder (Cambridge University Press, Cambridge, 1979).

[59] G. Jacucci and I.R. McDonald, Physica 80A, 607 (1975).

[60] K. W. Kehr, K. Binder, and S. Reulein, Phys. Rev. B 39, 4891 (1989).

[61] J. Horbach and W. Kob, Phys. Rev. B 64, 041503 (2001).

[62] L. Landau and E. Lifshitz, Fluid Mechanics (Pergamon Press, Oxford, 1987). 


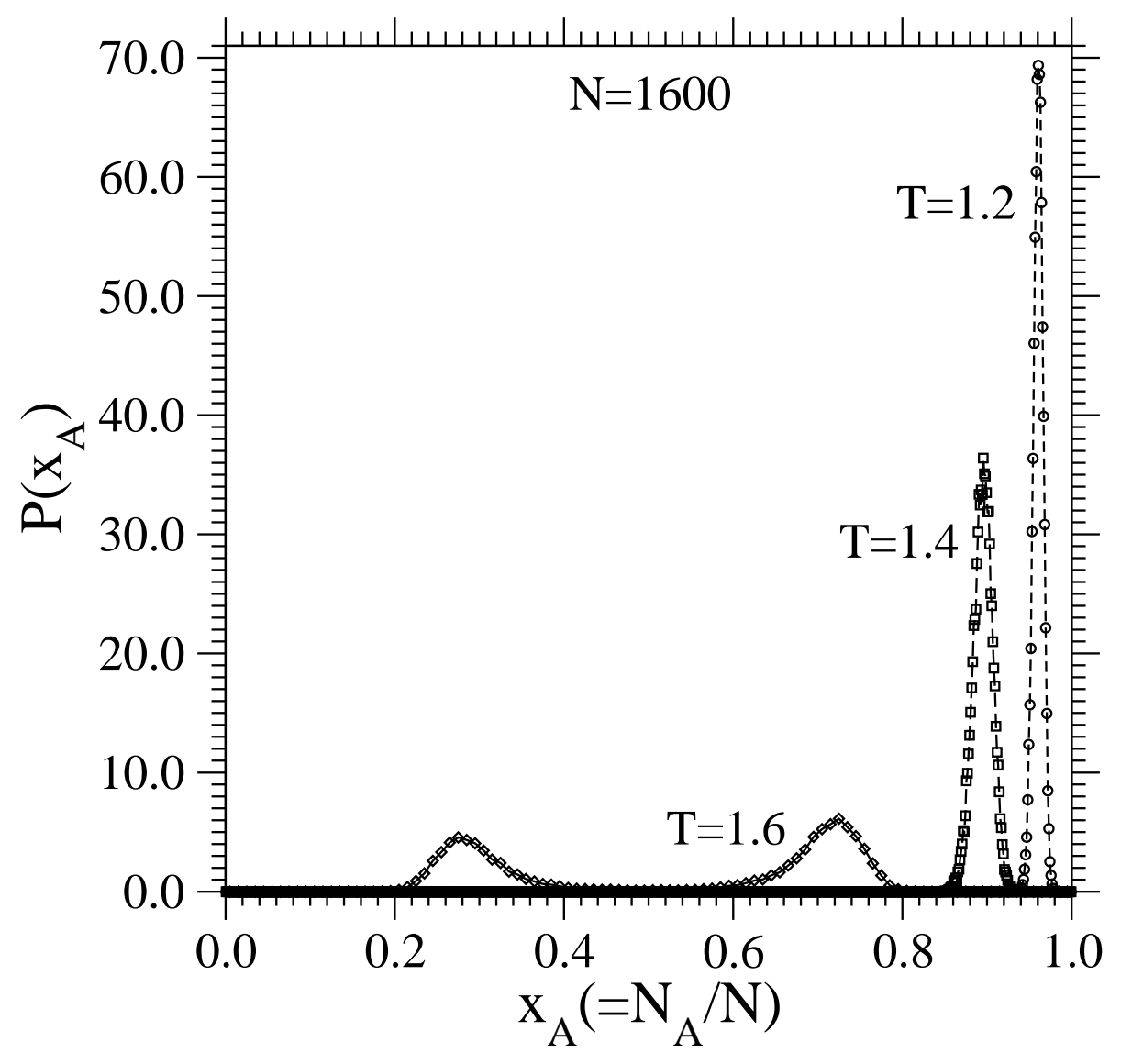

Figure 1: Distribution function $P\left(x_{\mathrm{A}}\right)$ for the relative concentration $x_{\mathrm{A}}=$ $N_{\mathrm{A}} / N$ of A particles for $N=1600$ particles at density $\rho=1$ and the three indicated temperatures. 


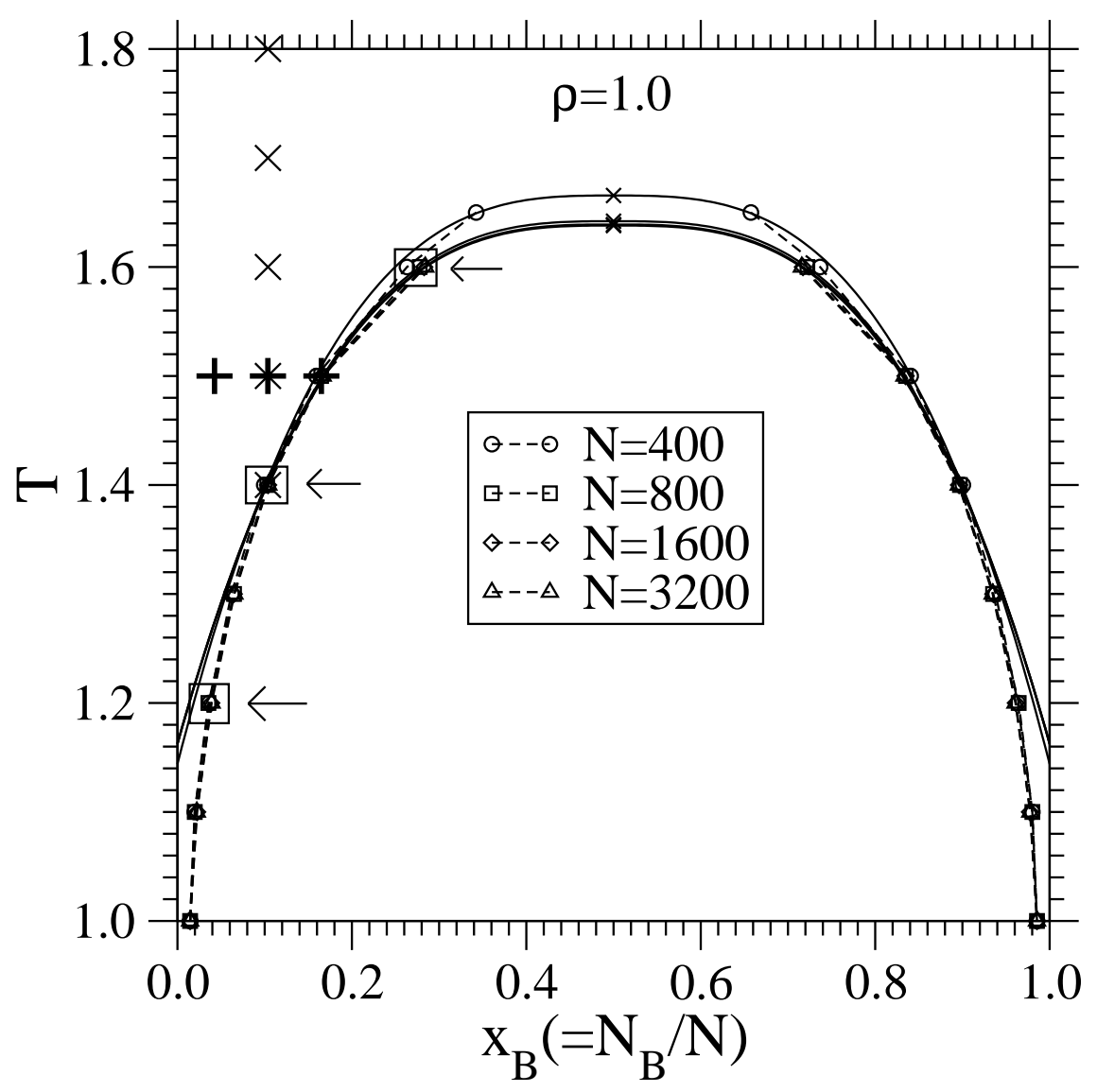

Figure 2: Phase diagram of the symmetrical binary Lennard-Jones mixture in the plane of variables temperature $T$ and concentration $x_{\mathrm{B}}=N_{\mathrm{B}} / N$, for density $\rho=1.0$ and four choices of $N$, as indicated. Crosses and plus symbols indicate paths where the structure and dynamics is studied at constant concentration $x_{\mathrm{B}}=0.10375$ as a function of temperature and at constant temperature $T=1.5$ as a function of concentration $x_{\mathrm{B}}$, respectively (see Secs. 4 and 5.2). Squares with arrows show states at the coexistence curve, which will be analyzed in detail in Secs. 4 and 5.1. The full curves show power law fits with Eq. (4). For $N=400$ (thin curve) one obtains $T_{c} \approx 1.666 \pm 0.005$, while for all larger values of $N$ the result is $T_{c} \approx 1.638 \pm 0.005$. The broken curves are guides to the eye only. The crosses at $x_{\mathrm{B}}=1 / 2$ mark the estimates for $T_{c}$ that result for the various choices of $N$. 

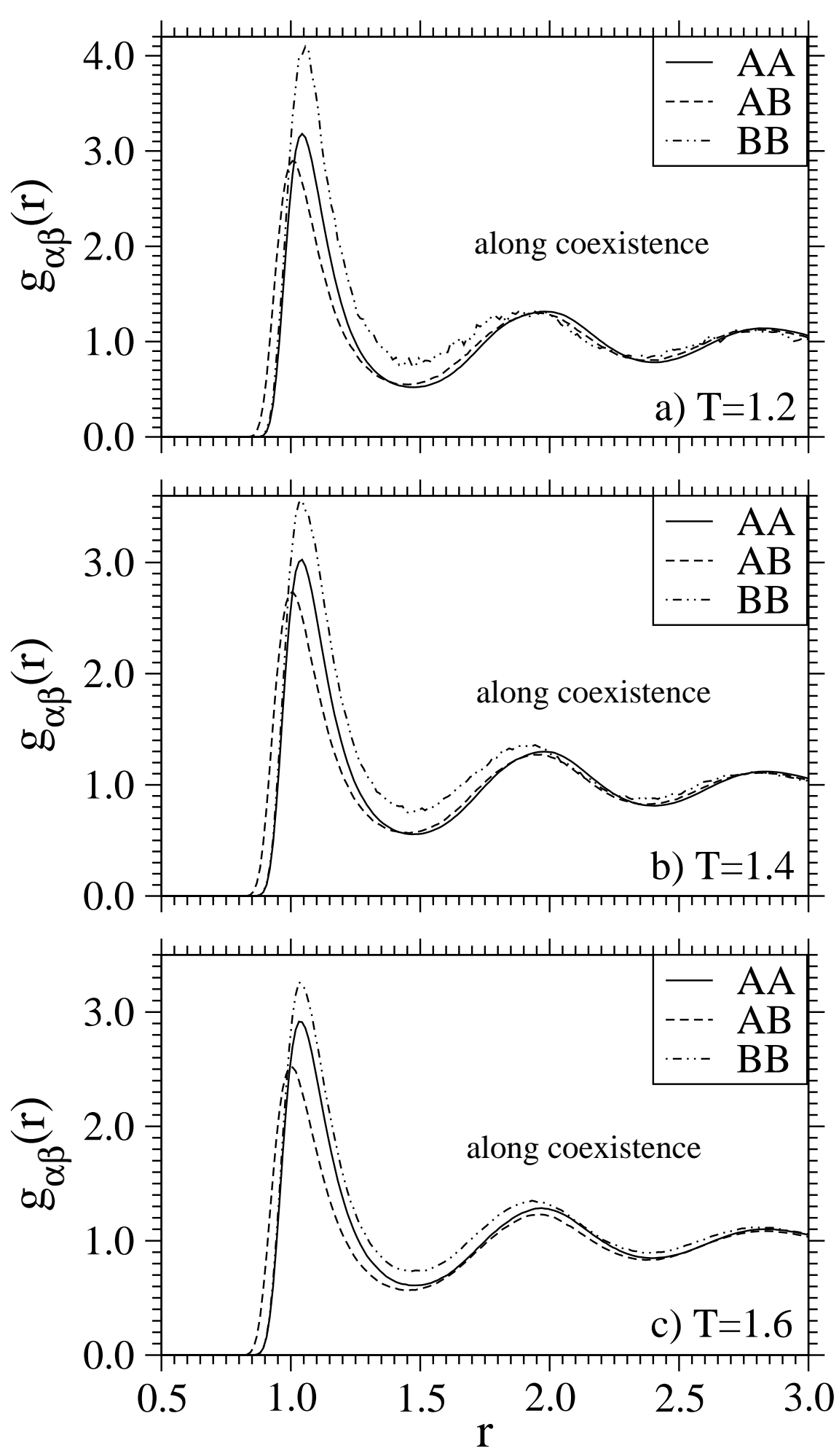

Figure 3: Radial distribution functions $g_{\mathrm{AA}}(r)$ (solid lines), $g_{\mathrm{AB}}(r)$ (dashed lines), and $g_{\mathrm{BB}}(r)$ (dashed-dotted lines) along the A-rich part of the coexistence curve (Fig. 2) plotted vs. $r$ at the temperatures a) $T=1.2$, b) $T=1.4$, and c) $T=1.6$. 


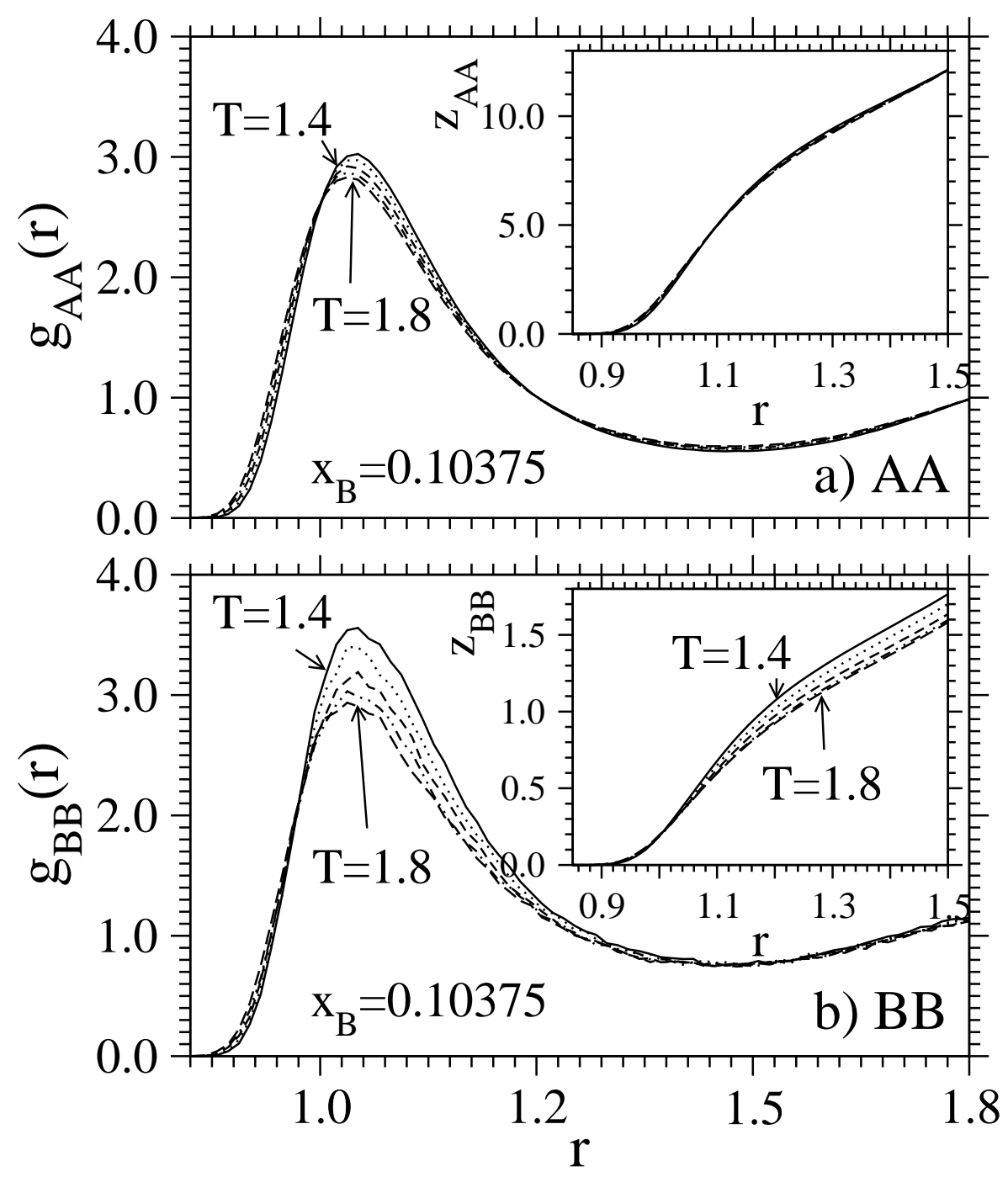

Figure 4: Radial distribution functions $g_{\mathrm{AA}}(r)$, part a), and $g_{\mathrm{BB}}(r)$, part b), for the temperatures $T=1.4,1.5,1.6,1.7$, and 1.8 at the concentration $x_{\mathrm{B}}=$ 0.10375 . The insets show the corresponding coordination numbers $z_{\alpha \alpha} \quad \alpha \in$ $[\mathrm{A}, \mathrm{B}]$ as a function of $r$. 


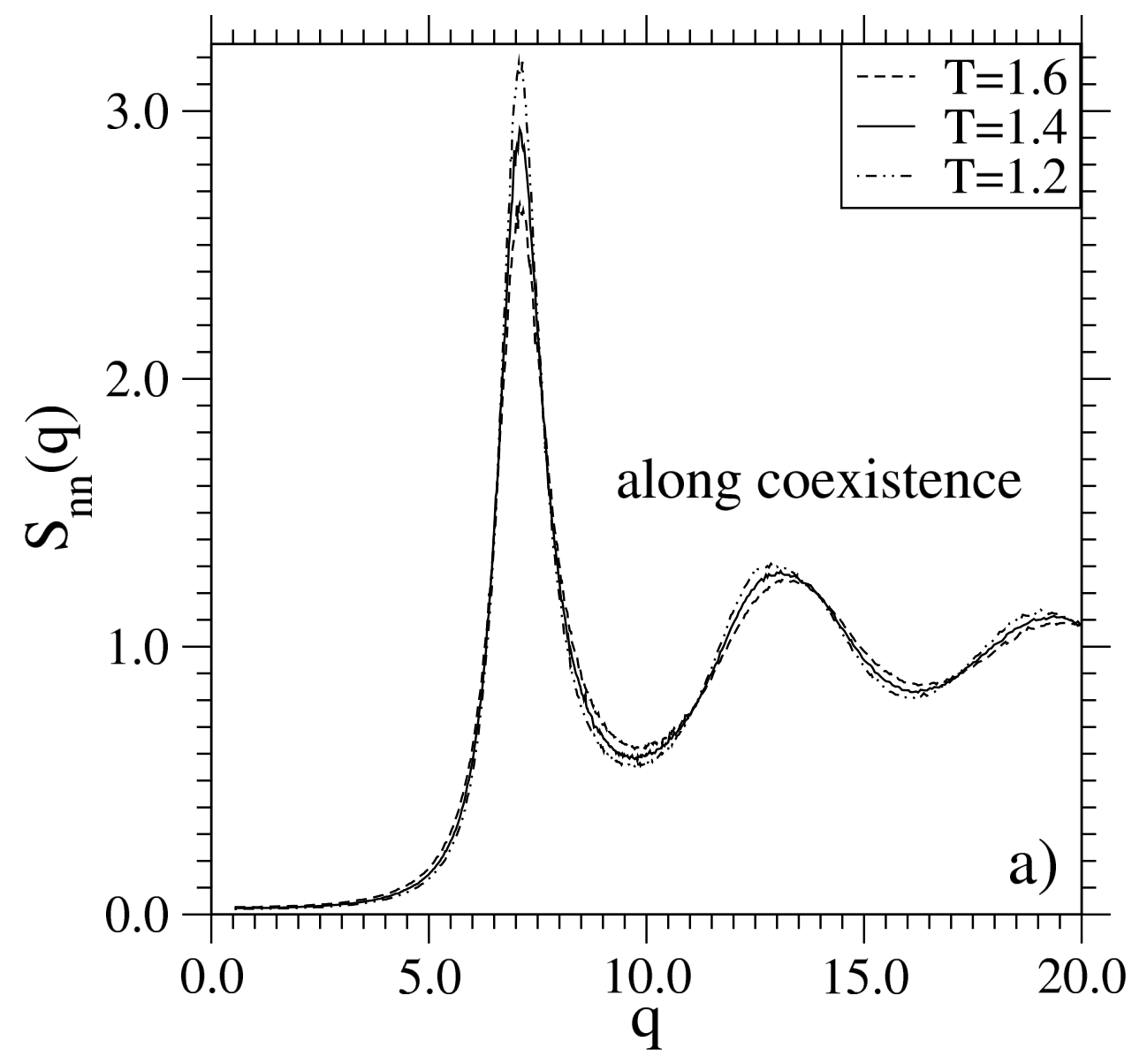



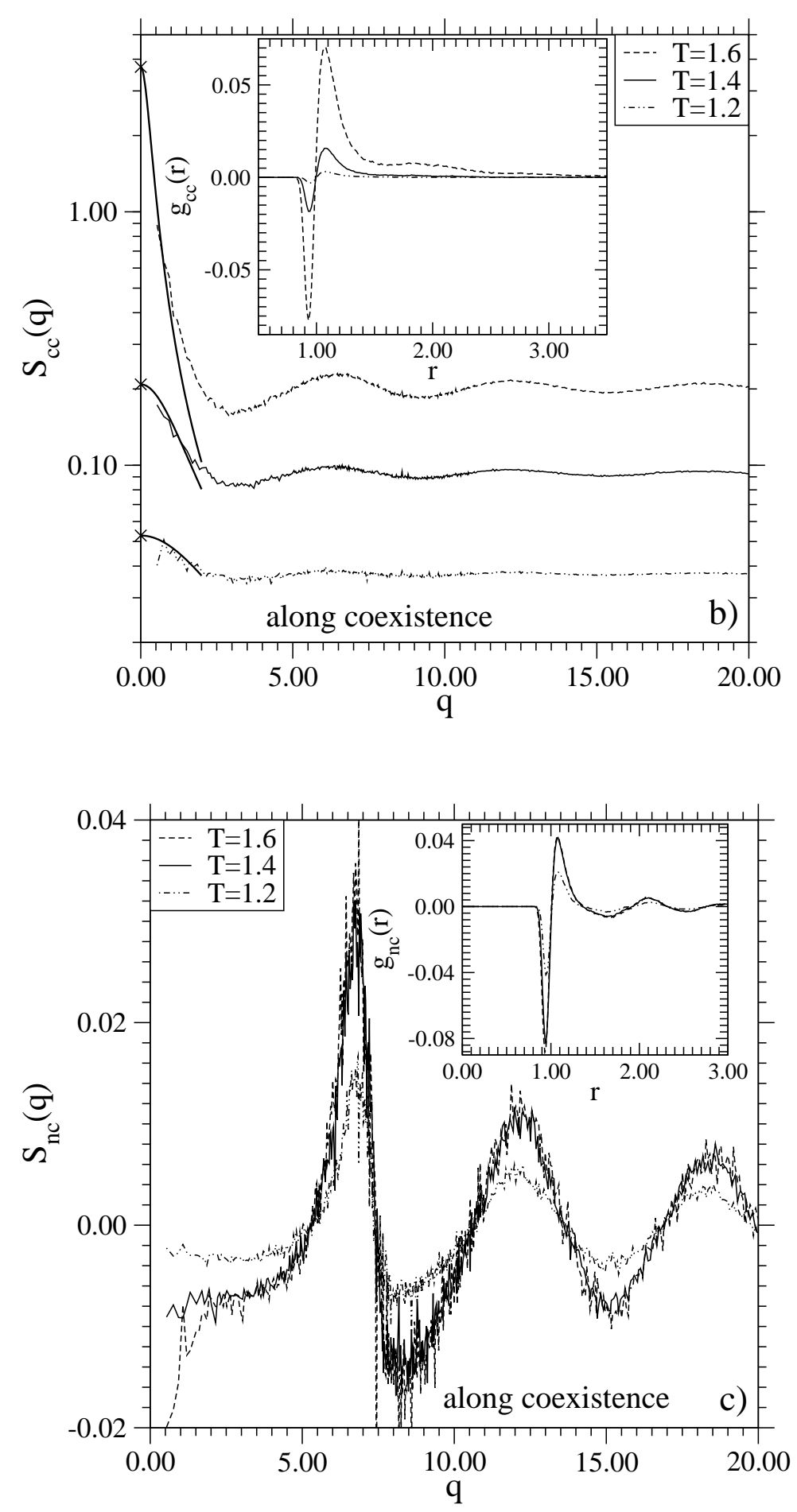

Figure 5: Different structure factors plotted vs. $q$ for the three indicated temperatures along the coexistence curve. a) $S_{n n}(q)$, b) $S_{c c}(q)$ (the bold solid lines are fits with Eq. 14 the crosses are estimated of $S_{c c}(q=0)$ as obtained from Eqs. (3) and (14)), and c) $S_{n c}(q)$. Note that a logarithmic scale is used on the ordinate of part b). The insets in b) and c) show the corresponding functions in real space. 

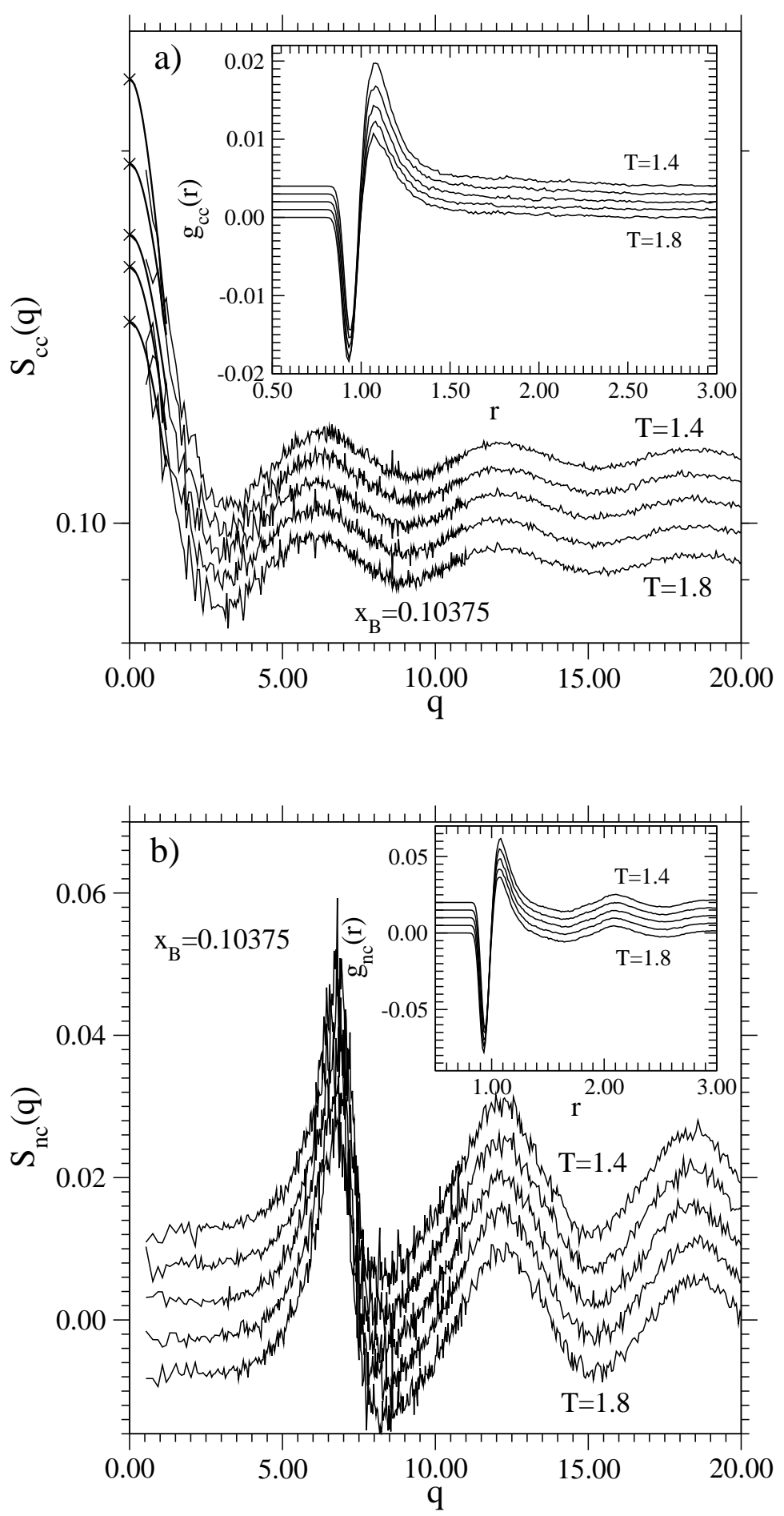

Figure 6: Structure factors $S_{c c}(q)$, part a), and $S_{n c}(q)$, part b), plotted vs. $q$, for the temperatures $T=1.4,1.5,1.6,1.7$, and 1.8 (from top to bottom), at the concentration $x_{\mathrm{B}}=0.10375$. For $S_{c c}$ again a logarithmic scale is used. The curves are overshifted relative to each other by an amount $\Delta=0.005(T=1.8$ is the unshifted curve). The crosses in part a) are estimates of $S_{c c}(q=0)$ as in Fig. 50 and the bold solid lines are fits with Eq. 14. The insets show the corresponding functions in real space (also overshifted to each other by an amount $\Delta=0.001$ in part a) and $\Delta=0.005$ in part b), again $T=1.8$ is the unshifted temperature). 

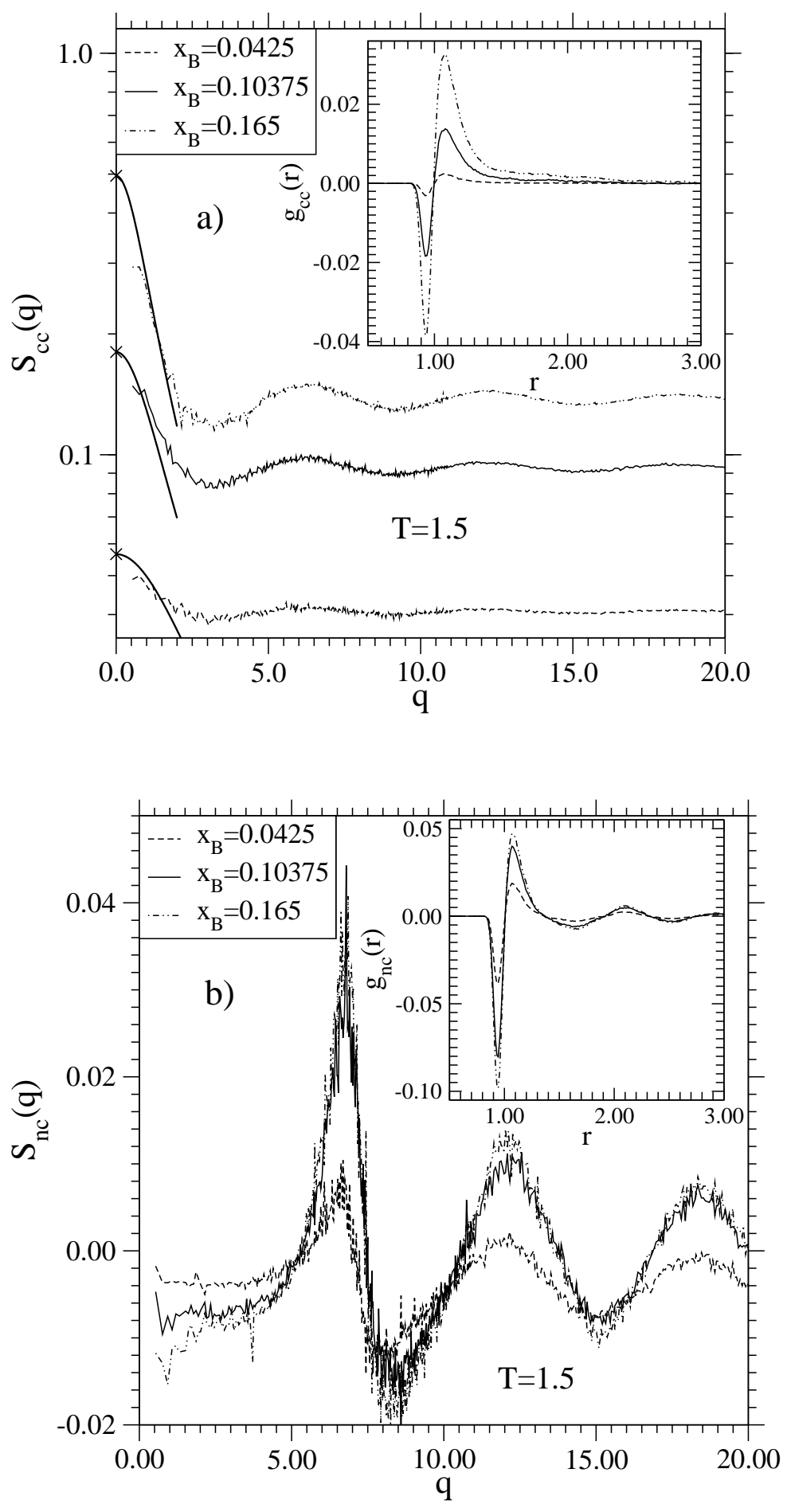

Figure 7: Same as Fig. [6] but at constant temperature $T=1.5$ and three concentrations as shown (note that all the curves are unshifted). 


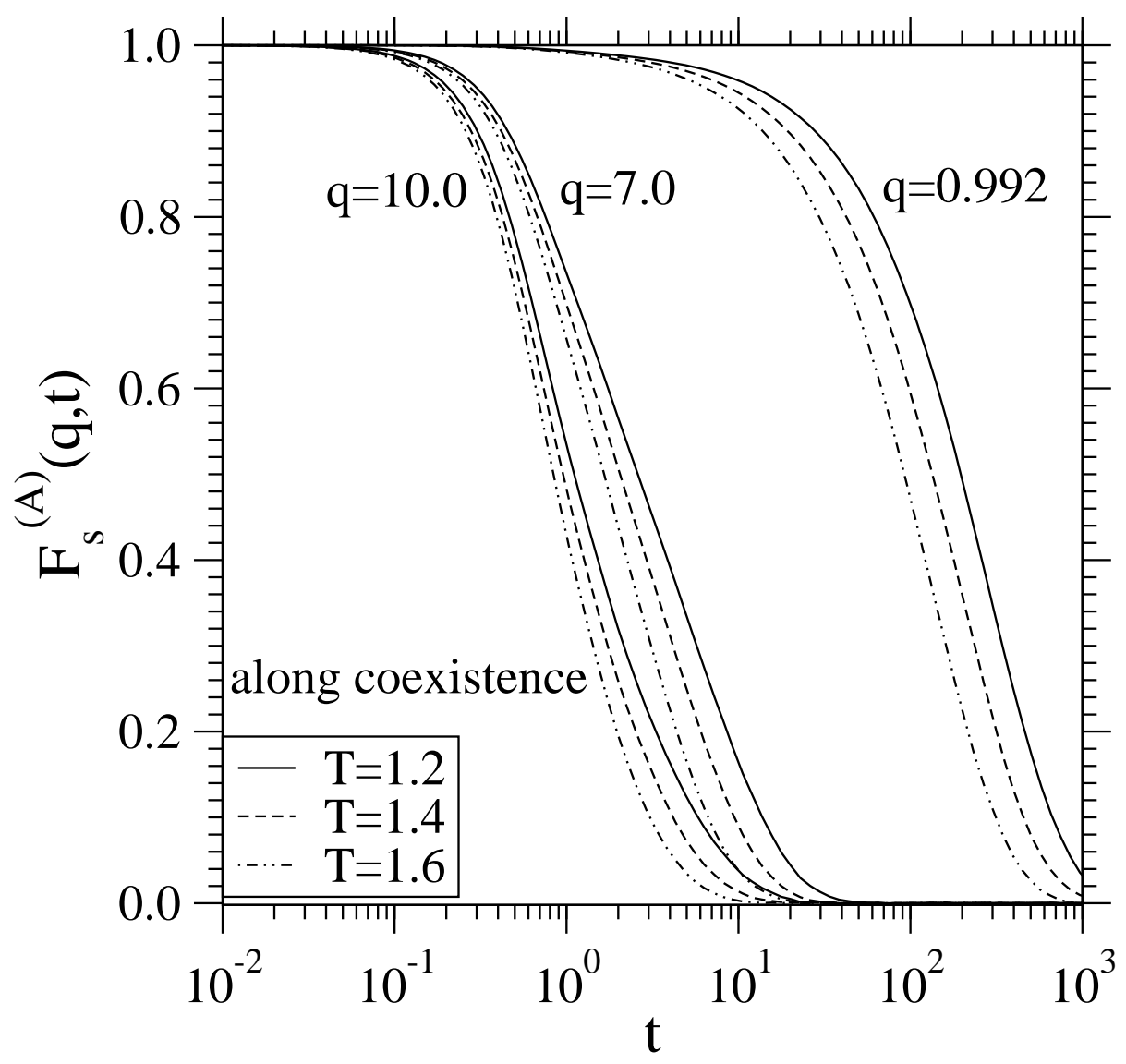

Figure 8: Incoherent intermediate scattering function of A particles plotted versus time (note the logarithmic scale of time) for the three temperatures $T=1.2$ (solid lines), $T=1.4$ (dashed lines), and $T=1.6$ (dashed-dotted lines) along the coexistence curve for three different values of $q$, namely: $q=0.992$, 7, 10 (from right to left). 


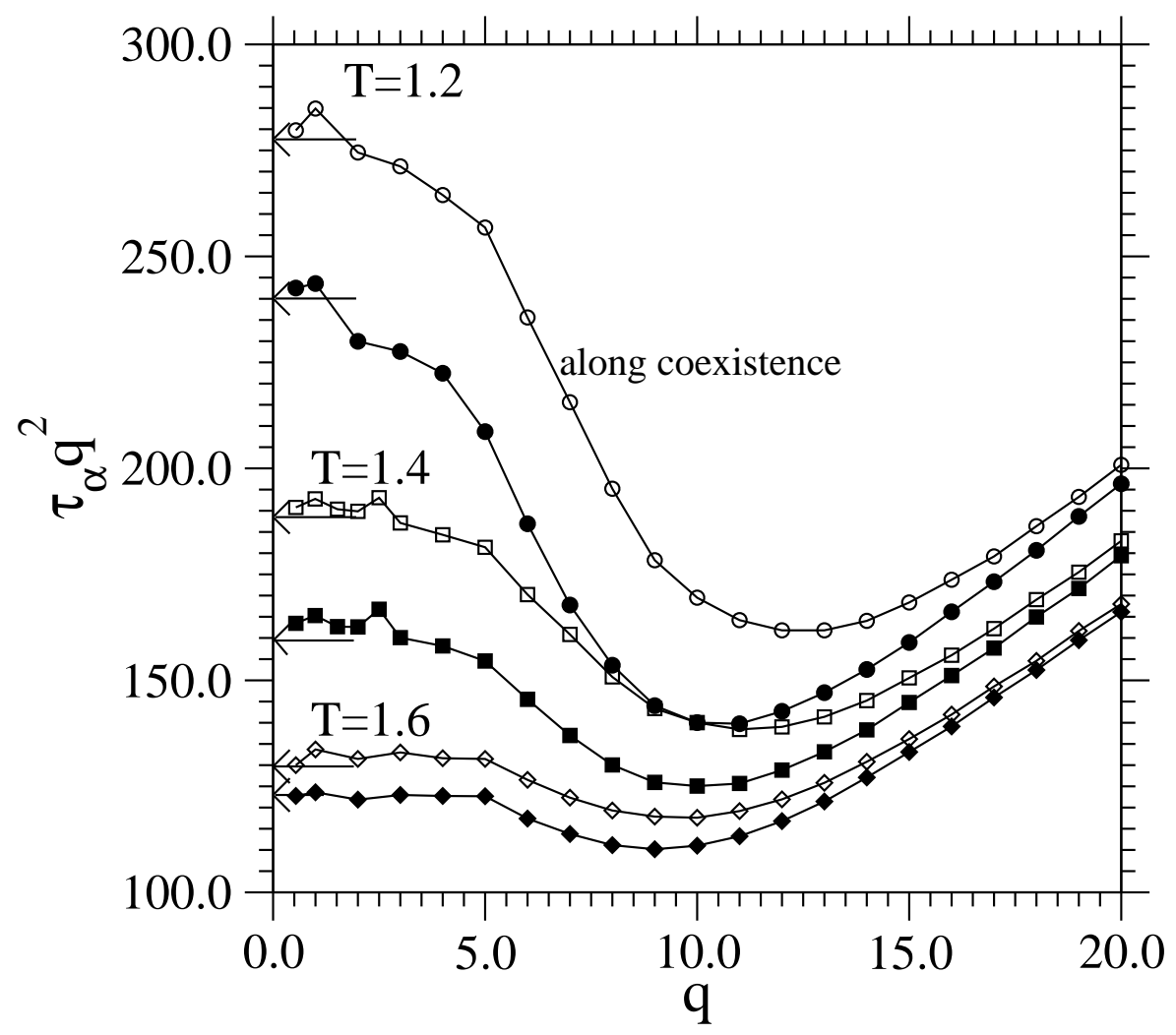

Figure 9: Plot of scaled relaxation time $\tau(q) q^{2}$ vs. $q$, for the A-particles (open symbols) and the B-particles (filled symbols) for three temperatures along the A-rich branch of the coexistence curve. The arrows indicate the value of the corresponding inverse self-diffusion constants. 

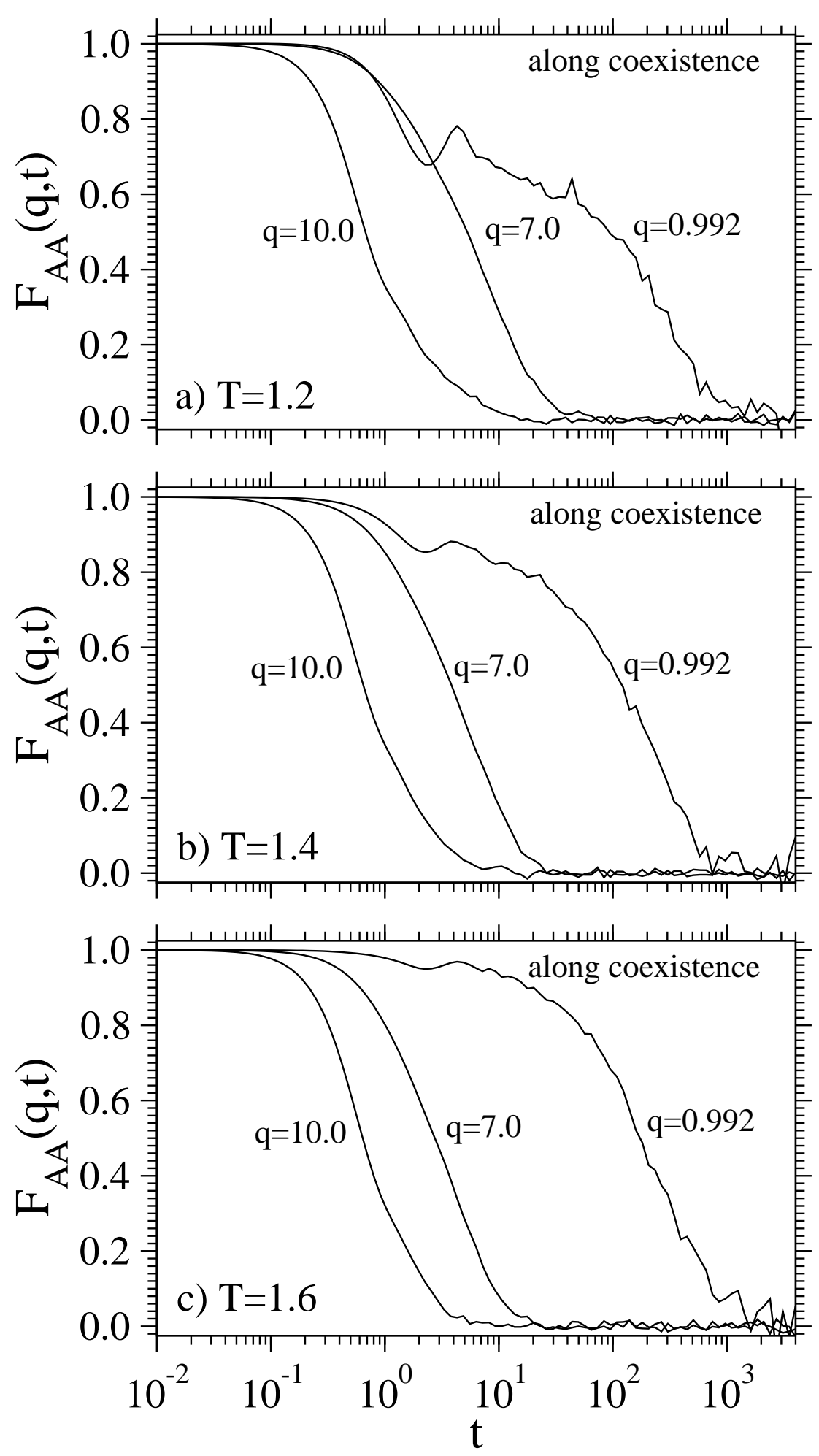

Figure 10: Coherent intermediate scattering function $F_{\mathrm{AA}}(q, t)$ of A-particles plotted vs. time (note logarithmic scale of time) for three temperatures and concentrations given from the A-rich part of the coexistence curve. Three temperatures are shown, $T=1.2$, part a), $T=1.4$, part b), and $T=1.6$, part c). Three wavenumbers $q$ are included as indicated. 

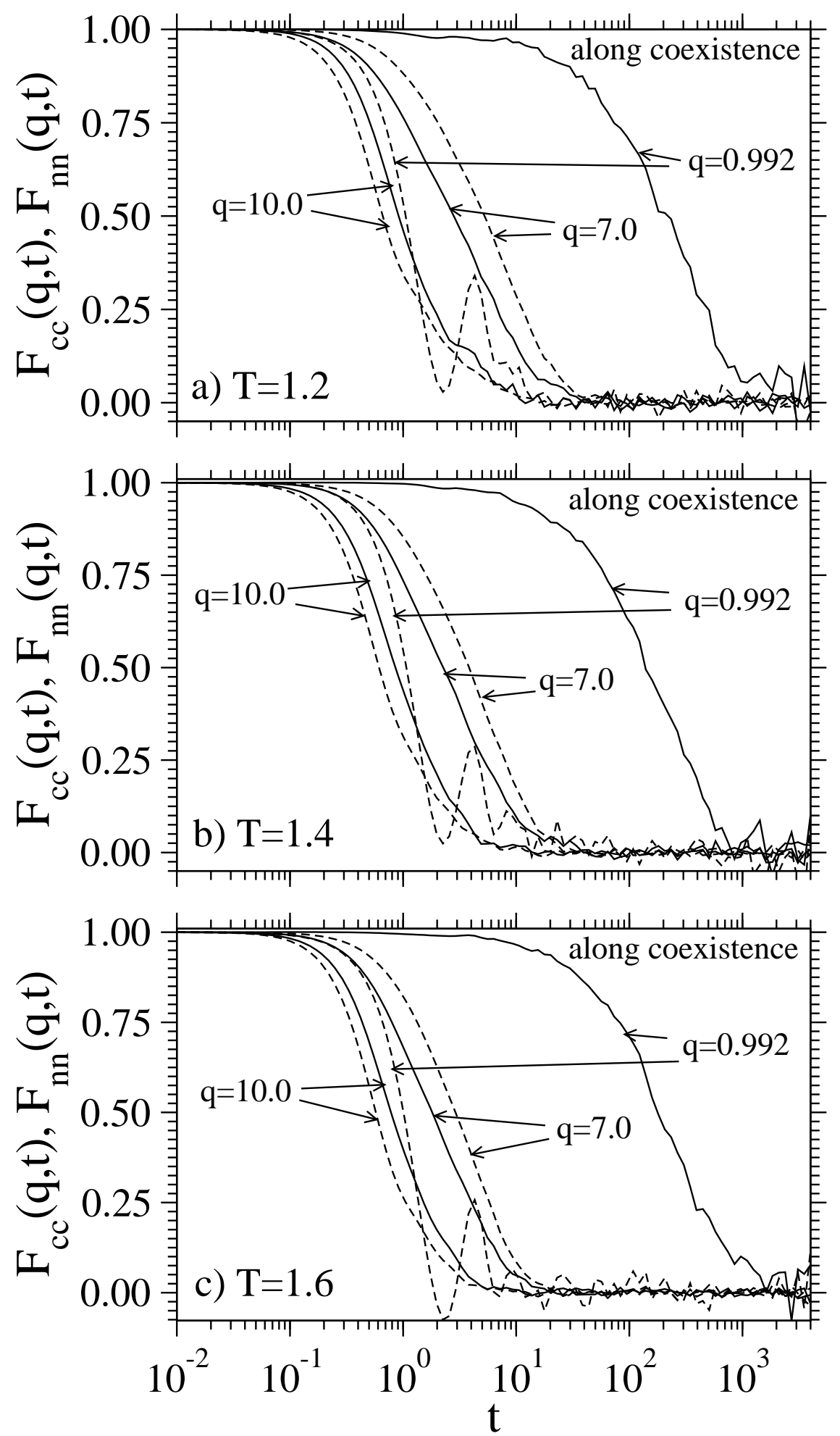

Figure 11: Same as Fig. 10 but for $F_{c c}(q, t)$ (solid lines) and $F_{n n}(q, t)$ (dashed lines). 


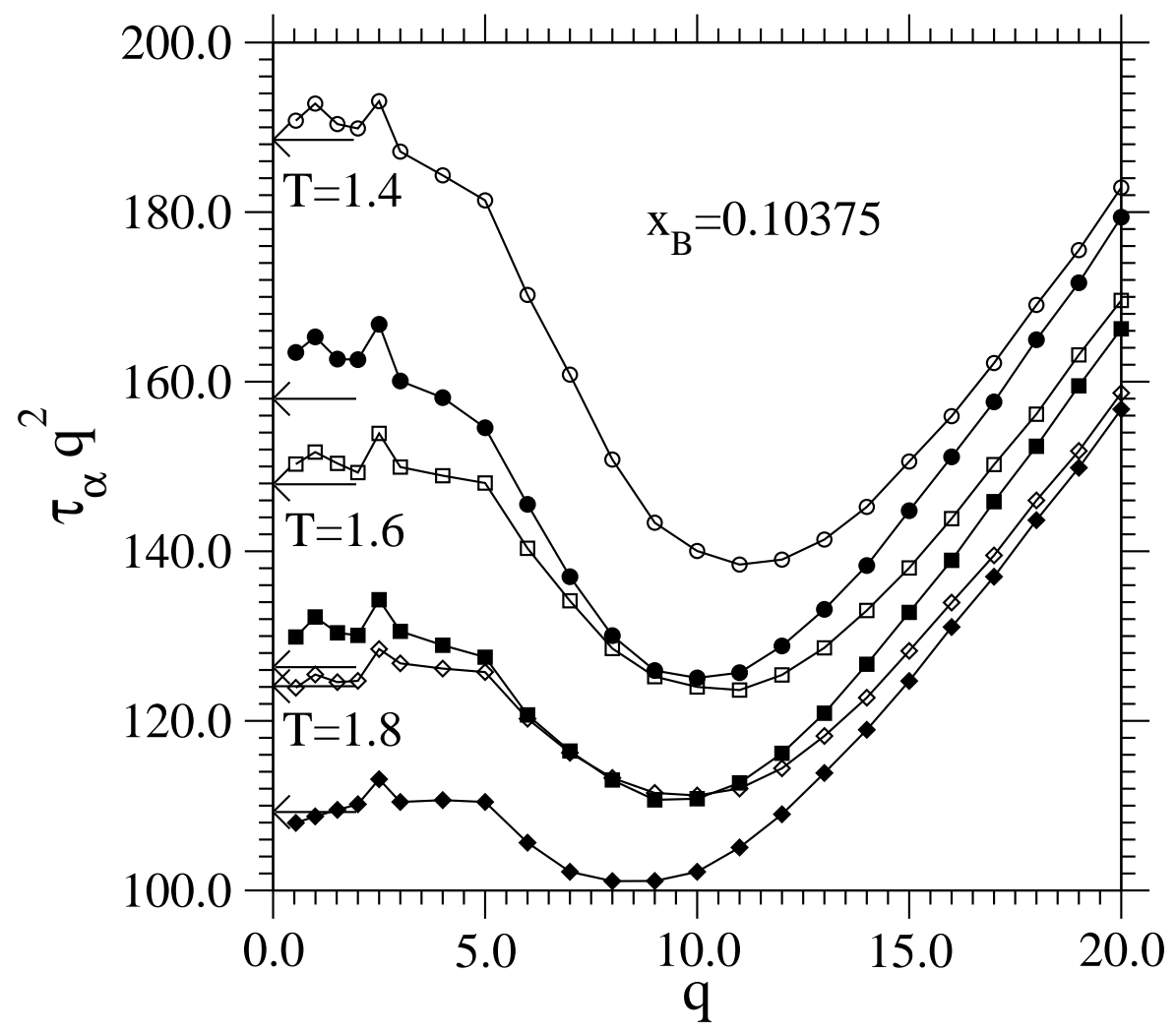

Figure 12: Scaled relaxation times $\tau_{\mathrm{A}}(q) q^{2}$ for the A-particles (open symbols) and $\tau_{\mathrm{B}}(q) q^{2}$ for the B-particles (filled symbols) plotted vs. wavenumber $q$. Three temperatures at constant $x_{\mathrm{B}}=0.10375$ are shown. The arrows indicate the values of the corresponding inverse diffusion constants, as extracted from the mean square displacements via the Einstein relation. 


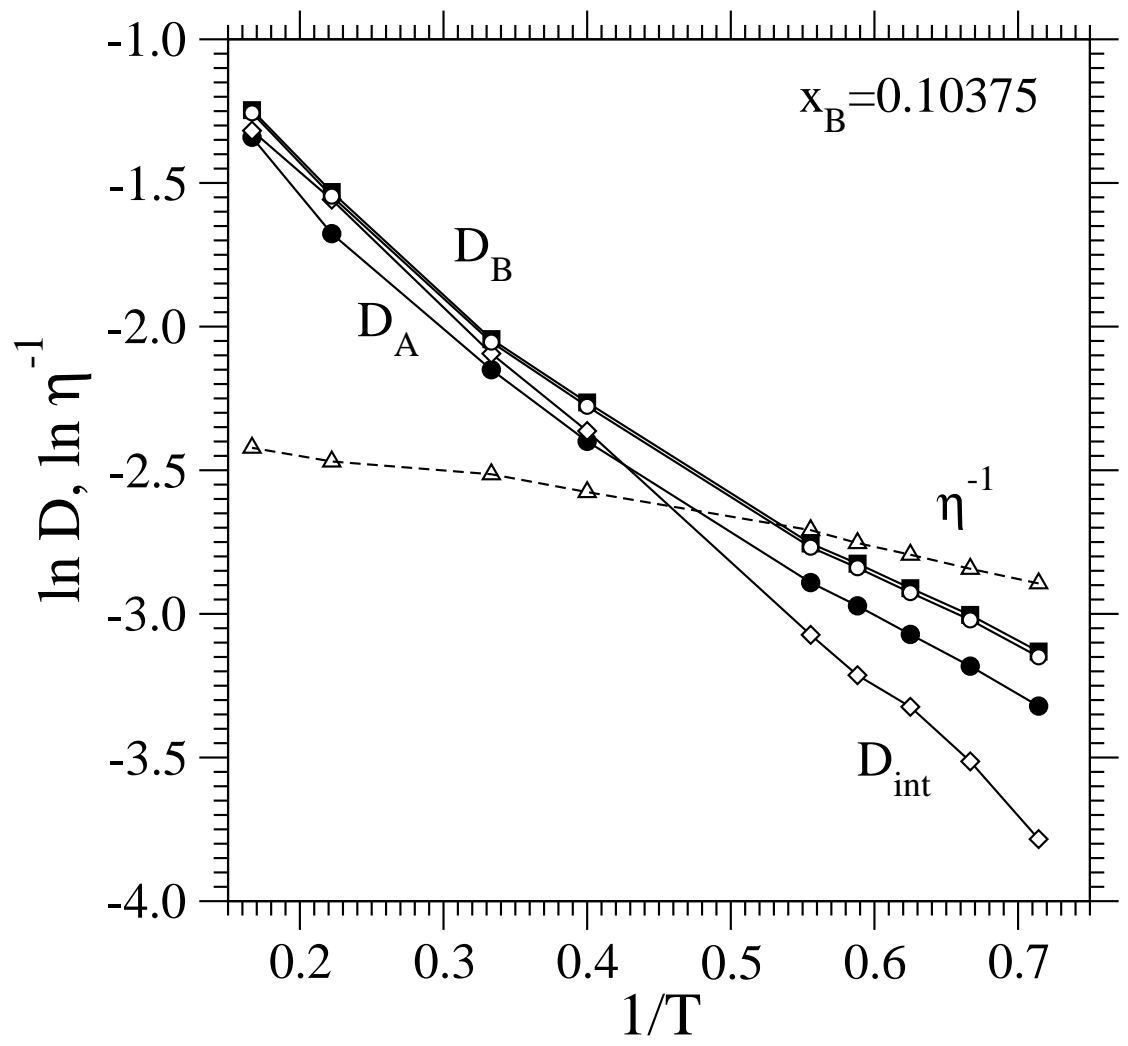

Figure 13: Logarithm of the self-diffusion constants (filled symbols) and the interdiffusion constant (open diamonds) as well as of the inverse viscosity (open triangles) plotted vs. $1 / T$ for a wide range of temperatures for the mixture at constant concentration $x_{\mathrm{B}}=0.10375$. Also plotted is $D_{\text {int }}$ as calculated from Eq. (28) (open circles). The viscosity is multiplied by a factor of 3 . The lines are guides to the eye. 


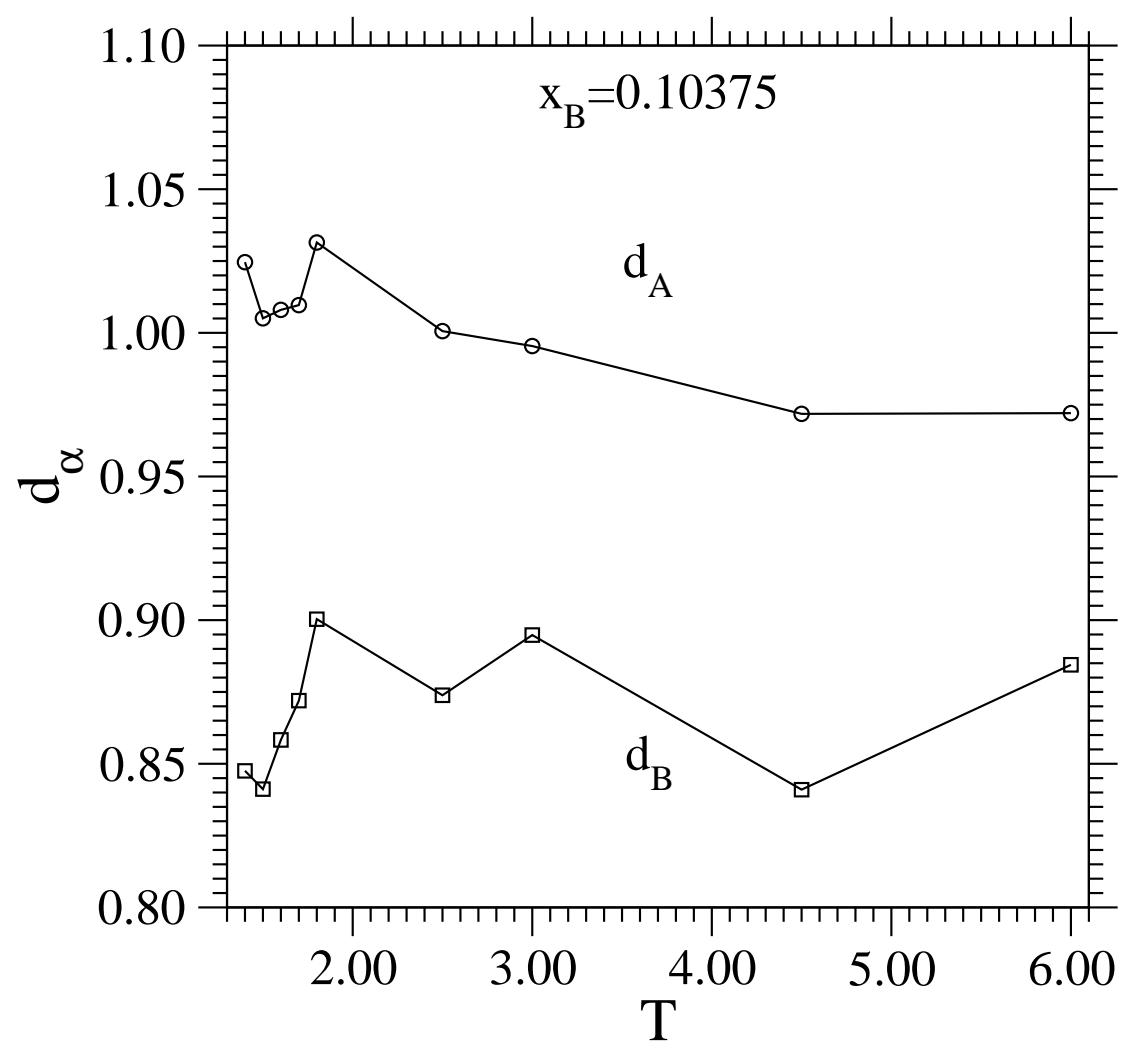

Figure 14: Stokes-Einstein diameters $d_{\mathrm{A}}, d_{\mathrm{B}}$ of the diffusing particles plotted vs. temperature, for the mixture at constant concentration $x_{\mathrm{B}}=0.10375$. 